BNL-79662-2007-IR

\title{
Evaluation of Neutron Cross Sections for a Complete Set of Nd Isotopes
}

\author{
Hyeong II Kim a, M. Herman b, S. F. Mughabghab b, \\ P. Oblozinsky b, D. Rochman b, Young-Ouk Lee a \\ aNuclear Data Evaluation Laboratory, Korea Atomic Energy Research Institute \\ 150 Yuseong, Daejeon 305-353, Korea \\ bNational Nuclear Data Center, Brookhaven National Laboratory \\ Upton, NY 11973-5000, USA \\ 12 December 2007
}

\author{
Energy Sciences \& Technology Department \\ National Nuclear Data Center \\ Brookhaven National Laboratory \\ P.O. Box 5000 \\ Upton, NY 11973-5000 \\ www.bnl.gov
}

\footnotetext{
Notice: This manuscript has been authored by employees of Brookhaven Science Associates, LLC under Contract No. DE-AC02-98CH10886 with the U.S. Department of Energy. The publisher by accepting the manuscript for publication acknowledges that the United States Government retains a non-exclusive, paid-up, irrevocable, world-wide license to publish or reproduce the published form of this manuscript, or allow others to do so, for United States Government purposes.
} 


\section{DISCLAIMER}

This report was prepared as an account of work sponsored by an agency of the United States Government. Neither the United States Government nor any agency thereof, nor any of their employees, nor any of their contractors, subcontractors, or their employees, makes any warranty, express or implied, or assumes any legal liability or responsibility for the accuracy, completeness, or any third party's use or the results of such use of any information, apparatus, product, or process disclosed, or represents that its use would not infringe privately owned rights. Reference herein to any specific commercial product, process, or service by trade name, trademark, manufacturer, or otherwise, does not necessarily constitute or imply its endorsement, recommendation, or favoring by the United States Government or any agency thereof or its contractors or subcontractors. The views and opinions of authors expressed herein do not necessarily state or reflect those of the United States Government or any agency thereof. 


\title{
Evaluation of Neutron Cross Sections for a Complete Set of Nd Isotopes
}

\author{
Hyeong Il Kim ${ }^{\text {a,* }}$, M. Herman ${ }^{\text {b }}$, S. F. Mughabghab ${ }^{\text {, }}$ \\ P. Obložinský ${ }^{b}$, D. Rochman ${ }^{b}$, Young-Ouk Lee ${ }^{a}$ \\ ${ }^{a}$ Nuclear Data Evaluation Laboratory, Korea Atomic Energy Research Institute \\ 150 Yuseong, Daejeon 305-353, Korea \\ ${ }^{\mathrm{b}}$ National Nuclear Data Center, Brookhaven National Laboratory \\ Upton, NY 11973-5000, USA
}

\begin{abstract}
Neutron cross sections for a complete set of Nd isotopes, ${ }^{142,143,144,145,146,147,148,150} \mathrm{Nd}$, were evaluated in the incident energy range from $10^{-5} \mathrm{eV}$ to $20 \mathrm{MeV}$. In the low energy region, including thermal and resolved resonances, our evaluations are based on the latest data published in the Atlas of Neutron Resonances. In the unresolved resonance region we performed additional evaluation by using the averages of the resolved resonances and adjusting them to the experimental data. In the fast neutron region, we used the nuclear reaction model code EMPIRE-2.19 validated against the experimental data. The results are compared to the existing nuclear data libraries, including ENDF/B-VI.8, JENDL-3.3 and JEFF-3.1, and to the available experimental data. The new evaluations are suitable for neutron transport calculations and they were adopted by the new evaluated nuclear data file of the United States, ENDF/B-VII.0, released in December 2006.
\end{abstract}

Key words: Neutron-induced reactions, Neutron resonances, Evaluations, Optical model, Hauser-Feshbach model, Direct and preequilibrium reactions, Neodymium, EMPIRE-2.19, ENDF/B-VII.0

PACS: 24.10.-i, 24.10.Eq, 24.60.-k, 24.60.Dr, 24.60.Gv, 25.40.-h, 25.40.Fq

* Corresponding author.

Email addresses: hikim@kaeri.re.kr (Hyeong Il Kim), mwherman@bnl.gov (M. Herman), mugabgab@bnl.gov (S. F. Mughabghab), oblozinsky@bnl.gov (P.

Obložinský), rochman@nrg-nl.com, present address NRG, PO Box 25, 1755 ZG Petten, The Netherlands (D. Rochman), yolee@kaeri.re.kr (Young-Ouk Lee). 


\section{Introduction}

Fission products, broadly defined as nuclei with $\mathrm{Z}=31-68$, accumulate in an operating reactor as the fuel undergoes fission. Many of them have large neutron absorption cross sections in thermal, resonance and keV energy ranges. Furthermore, they release radiation and heat after shutdown as well as during reactor operation. Consequently, spent fuel is highly radioactive when removed from reactor and it must be cooled by being stored prior to processing or disposal. Accordingly, neutron cross section data on fission products play an important role not only in the design of an extended burn-up core and fast reactors, but also in the study of the back-end fuel cycle and the criticality analysis of spent fuel.

Many fission product evaluations in the U.S. ENDF/B-VI library were not revised for a long period of time, often 30-35 years, making the need for new evaluations a pressing issue. As a part of the effort to rectify this situation, a collaborative BNL - KAERI project was initiated with the aim to produce improved evaluations for the most important fission products. The project produced new files for 32 fission products [1], including a complete set of $\mathrm{Nd}$ isotopes. All these evaluations were adopted by the new U.S. evaluated library, ENDF/B-VII.0, released in December 2006 [2].

The priorities for the fission products were identified by considering fission yields and neutron capture cross sections. Following the analysis by DeHart, ORNL, in 1995 [3], ${ }^{143,145} \mathrm{Nd}$ ranked 2 and 11 on the list of possible fission products, respectively, for initial enrichment of $4.5 \%{ }^{235} \mathrm{U}$, burnup of $50 \mathrm{GWD} / \mathrm{MTU}$, and a 5-year cooling period. The present exercise extends to all remaining isotopes of neodymium, including stable ${ }^{142,144,146,148,150} \mathrm{Nd}$ and radioactive ${ }^{147} \mathrm{Nd}$, to take full advantage of the simultaneous evaluation of the complete isotopic chain with modern evaluation tools and procedures.

The previous U.S. evaluations for Nd were performed in 1980 and did not take into account more recent measurements. The BNL-KAERI collaborative project first addressed the evaluations of the resonance region for 19 priority fission products including ${ }^{143,145} \mathrm{Nd}$ that were adopted by ENDF/B-VI.8 library in 2001 [4]. Then, the present work revisited ${ }^{143,145} \mathrm{Nd}$, added fast neutron region and extended the evaluation effort to all remaining $\mathrm{Nd}$ isotopes in the full energy range from $10^{-5} \mathrm{eV}$ to $20 \mathrm{MeV}$.

Our evaluations take advantage of a number of recent developments combined into powerful evaluation tool that, by far, exceeds previous capabilities. These developments combine a number of codes, methods, databases and computer power into a single product of considerable power and performance.

The paper is organized as follows. In Chapter 2 we review the status of avail- 
able evaluations and the status of new experimental data since 1980. In Chapter 3 we describe the evaluation methodology. Then, we proceed with actual evaluations, Chapter 4 is devoted to the low energy region, followed by Chapter 5 describing the fast neutron region. Conclusions are drawn in Chapter 6 .

\section{Status of evaluations and measurements}

Our results are always compared with the existing evaluated nuclear data libraries, ENDF/B-VI.8, JENDL-3.3 and JEFF-3.1. The Nd evaluations in these libraries can be summarized as follows:

- ENDF/B-VI.8 (released in 2001)

- General: ENDF/B-V was converted to ENDF-6 format to create initial version of the ENDF/B-VI library in 1990. The evaluations by Schenter et al. of ${ }^{142,147} \mathrm{Nd}$ were completed in 1974 and those of ${ }^{143,144,145,146,148,150} \mathrm{Nd}$ in 1980 .

- Resonance parameters: Parameters recommended by Mughabghab et al. [5] were adopted, those of ${ }^{143,145} \mathrm{Nd}$ were modified by J. Chang and S.F. Mughabghab in 1999.

- Fast neutron region: Theoretical calculations were performed using the optical model potential (OMP) of Moldauer [6], COMNUC-3 code for inelastic and capture, and NCAP code for capture. Angular distributions of neutrons were assumed to be isotropic except for the elastic scattering.

- JEFF-3.1 (released in 2005)

- General: JEFF-3.1 and JEFF-3.0 are the same as JEF-2.2 which adopted old ENDF/B-V evaluations for ${ }^{142,147,150} \mathrm{Nd}$, the ENEA evaluation (1979) for ${ }^{143} \mathrm{Nd}$, and RCN-3 evaluation (1978) for ${ }^{144,145,146,148} \mathrm{Nd}$.

- Resonance parameters: The parameters for ${ }^{142,143,147,150} \mathrm{Nd}$ were updated in 1980 and for ${ }^{144,145} \mathrm{Nd}$ in 1986 using the data from Ref. [7]. The resonance region of ${ }^{146,148} \mathrm{Nd}$, based on the data from Ref. [5], were corrected by inserting background cross sections in 1990.

- Fast neutron region: No detailed information is available. The charged particle emission cross sections were included in 1989 adopting REACECN-4.

- JENDL-3.3 (released in 2002)

- General: JENDL-3.3 is the same as JENDL-3.2 that propagated from JENDL-3.1. The evaluations of ${ }^{142,143,144,145,146,148,150} \mathrm{Nd}$ were made in 1984 for JENDL-2 and revised in 1990 for JENDL-3.1, while ${ }^{147} \mathrm{Nd}$ was newly evaluated in 1990. All evaluations were prepared by the JNDC Fission Products Nuclear Data working group.

- Resonance parameters: Resolved resonance parameters were based on the data of Tellier [8] and Musgrove et al. [9]. A negative resonance was added 
to reproduce the thermal capture cross sections of Ref. [7]. The resonance parameters of ${ }^{142,143,144,145} \mathrm{Nd}$ were updated in JENDL-3.2.

- Fast neutron region: Nuclear reaction model calculations for total, elastic and inelastic scattering, and capture cross sections were carried out with the statistical code CASTHY, direct inelastic with DWUCK-4, direct/semidirect capture with a simple formula [10], while other reaction channels were computed with preequilibrium and multi-step model code PEGASUS.

New, theory based, evaluation techniques make extensive use of model calculations with experimental data being critical for constraining model parameters. Such adjusted set of parameters is expected to perform reliably also for the reactions and/or isotopes for which no measurements are available. We searched for the experimental data in the experimental database CSISRS/EXFOR, and surveyed relevant journal articles and available reports. Since the last evaluation of the fast neutron region for the ENDF/B-VI.8, carried out in 1980, the following new cross section measurements on $\mathrm{Nd}$ isotopes were performed:

- (n,tot): Poenitz et al. [15], Wisshak et al. [16]

- $(\mathrm{n}, \gamma)$ : Wisshak et al. [16], Bokhovko et al. [17], Trofimov [18,19], Afzal Ansari et al. [20]

- (n,2n): Kasugai et al. [21], Gmuca et al. [22], An et al. [23]

- (n,p): Grallert et al. [24], Gmuca et al. [22], Sakane et al. [25], Kasugai et al. [21], Bari [26]

- (n, $\alpha$ ): Grallert et al. [24], Gmuca et al. [22], Bari [26], Koehler et al. [27], Sakane et al. [25], Majeddin et al. [28].

The results are summarized in Table 1. Although experimental data for many reaction channels are available, they are usually insufficient to fix model parameters unambiguously. For instance, total cross sections for many Nd isotopes were measured only in the low energy region while the fast neutron region remains unexplored, except for the single-energy points available for most of the isotopes, except for ${ }^{144} \mathrm{Nd}$. In order to work around the insufficient experimental data and constrain model parameters for all of the neodymium isotopes, we performed a simultaneous evaluation for the entire chain.

\section{Evaluation methodology}

Our evaluations are based on the Atlas-EMPIRE methodology developed over several recent years by the National Nuclear Data Center, BNL, with several external collaborators. This methodology covers both the low-energy region (thermal energy, resolved resonances, unresolved resonances) and the fast neutron energy region. 
Table 1

Summary of neutron-induced reaction channels for 8 neodymium isotopes for which experimental data are available. The isotopic abundances and the upper boundaries of the unresolved resonance region (URR) are also given.

\begin{tabular}{cccc}
\hline Isotope & $\begin{array}{c}\text { Experimental } \\
\text { data }\end{array}$ & $\begin{array}{c}\text { Abundance } \\
\%\end{array}$ & $\begin{array}{c}\text { URR upper limit } \\
\mathrm{keV}\end{array}$ \\
\hline${ }^{142} \mathrm{Nd}$ & tot, $e l, \gamma, 2 n, p, \alpha$ & 27.2 & 200.0 \\
${ }^{143} \mathrm{Nd}$ & tot, $\gamma, p, \alpha$ & 12.2 & 225.0 \\
${ }^{144} \mathrm{Nd}$ & tot, el, $\gamma, 2 n, \alpha$ & 23.8 & 250.0 \\
${ }^{145} \mathrm{Nd}$ & tot, $\gamma, p, \alpha$ & 8.3 & 67.69 \\
${ }^{146} \mathrm{Nd}$ & tot, el, $\gamma, 2 n, 3 n, p$ & 17.2 & 456.97 \\
${ }^{147} \mathrm{Nd}$ & - & 0.0 & 50.3 \\
${ }^{148} \mathrm{Nd}$ & tot, el, $\gamma, 2 n, 3 n, \alpha$ & 5.7 & 300.0 \\
${ }^{150} \mathrm{Nd}$ & tot, $e l, \gamma, 2 n, 3 n$ & 5.6 & 130.97 \\
${ }^{n a t} \mathrm{Nd}$ & tot $, e l, n^{\prime}$ & 100 & - \\
\hline
\end{tabular}

\subsection{Atlas-EMPIRE system}

In the low energy region, our methodology is based on the latest data published in the Atlas of Neutron Resonances [11]. This resource is used to infer both the thermal values and the resolved resonance parameters that are validated against the capture resonance integrals. The multi-level Breit-Wigner formalism is used to describe resonances. The resonance parameters are taken from the electronic version of the library that contains all data from the Atlas and is available locally at the NNDC.

In the unresolved resonance region (URR) one performs additional evaluation by using the average values of the resolved resonance parameters as the starting point. In cases where the resolved resonance parameters are not available, and suitable averages cannot be produced, one takes the average values from systematics described in the Atlas. Then, these initial average resonance parameters are adjusted to the experimental data. As a rule, the URR upper energy is defined by the first excited level of the target nucleus.

In the fast neutron region, our methodology is based on the nuclear reaction model code EMPIRE-2.19 $[12,13]$ with a consistent set of input parameters for a complete isotopic chain. EMPIRE is a modular system of codes consisting of many nuclear reaction models summarized below. The code is equipped with a powerful GUI interface, allowing an easy access to support libraries, the plotting package, as well the utility codes for formatting and checking. The 
two most important support libraries include the Reference Input Parameter Library [14], RIPL, and the library of experimental neutron cross sections CSISRS (also known as EXFOR).

In general, in EMPIRE calculations we use the RIPL-2 library for the initial set of model parameters. These parameters are properly adjusted to reproduce the available experimental data taken from the CSISRS library. Our evaluations cover cross sections for almost all reaction channels including photon production, energy spectra of emitted particles and their angular distributions, as well as recoils.

Then, the evaluated data are converted into ENDF-6 formatted files, checked by a set of the checking codes, processed with the processing code NJOY, and subjected to test runs with the Monte Carlo neutronics code MCNP to ensure that they can be used in transport calculations. For more details of this procedure see Ref. [2].

\subsection{Reaction model codes in EMPIRE}

All evaluations in the fast neutron region were performed with the new EMPIRE code system [13] that has been used for the first time to provide a number of consistent, complete evaluations to the evaluated nuclear data library. The nuclear reaction physics of EMPIRE is described elsewhere [12] and we are not going to repeat it here. Rather, we limit ourselves to a short summary of the codes included in the system.

EMPIRE calculates cross sections for all relevant reaction channels, angular distributions, exclusive and inclusive particle- and $\gamma$-spectra, double-differential cross sections, and spectra of recoils. The code observes angular momentum coupling (at least in the statistical decay part) and is, therefore, capable of detailed modeling of the $\gamma$-cascade providing $\gamma$ production spectra, intensities of discrete transitions, and isomeric cross sections. Nuclear reaction models included in the code can be classified into three major classes: (i) optical model and direct reactions (coupled-channels, CC, and distorted-wave Born approximation, DWBA), (ii) preequilibrium emission, and (iii) Hauser-Feshbach statistical decay. Inclusion of multiple preequilibrium processes allows us to cover incident energies ranging from the upper limit of resolved resonances up to about $150 \mathrm{MeV}$.

This modular code system integrates into a single system a number of important modules and specific nuclear reaction model codes. The list is given below, for more details including references we refer the reader to the recent extensive paper [12]: 
- Spherical and deformed Optical Model including coupled-channels (code ECIS03 by J. Raynal)

- Hauser-Feshbach Statistical Model including HRTW width fluctuation correction (code developed for EMPIRE by M. Herman)

- Quantum-mechanical Multi-step Direct Model in TUL formalism (codes ORION \& TRISTAN by H. Lenske), and Multi-step Compound Model in NVWY formalism (code developed for EMPIRE by M. Herman)

- Exciton Model with angular momentum coupling (code DEGAS by E. Běták and P. Obložinský) that represents a good approximation to the direct/semidirect capture model

- Exciton Model with Iwamoto-Harada cluster emission and Kalbach systematics for angular distribution (code PCROSS by R.Capote)

- Complete gamma-ray cascade after emission of each particle, including realistic treatment of discrete transitions

- Access to the Optical Model segment of the RIPL-2 library [14]

- Built-in input parameter files, such as masses, level density, discrete levels, fission barriers and gamma strength functions based on the RIPL-2 library

- Automatic retrieval of experimental data from the EXFOR/CSISRS library

- ENDF-6 formatting (code EMPEND by A. Trkov) coupled to graphical presentation capabilities (code ZVView by V. Zerkin) through the chain of preprocessing routines (PrePro codes by R. Cullen)

- ENDF checking codes (CHECKR, FIZCON, PSYCHE by C. Dunford)

- Support for the Los Alamos processing system (code NJOY by R. MacFarlane).

\section{Evaluation in the low energy region}

A statistical analysis of neutron resonances was used by S. Mughabghab, BNL, to produce the well known BNL-325 report. Its $5^{\text {th }}$ edition was published in 2006 as the "Atlas of Neutron Resonances: Resonance Parameters and Thermal Cross Sections, Z = 1 - 100" [11], hereafter referred to as the Atlas. This represents a considerable update to the 1981 [7] and 1984 [29] editions of BNL-325. These latest thermal values and resonance parameters provided a basis for the evaluations of six neodymium isotopes, ${ }^{142,144,146,147,148,150} \mathrm{Nd}$. The remaining evaluations, ${ }^{143,145} \mathrm{Nd}$ isotopes, were performed by J. Chang and S.F. Mughabghab in 1999 for ENDF/B-VI.8. In 2006, we reviewed these results, found good agreement with the Atlas and concluded that no changes are needed, though some other modifications were introduced as described below. 


\subsection{Thermal values and resolved resonances}

Accurate knowledge of the thermal neutron $(2200 \mathrm{~m} / \mathrm{sec}$ or $0.0253 \mathrm{eV})$ capture cross section is of paramount importance and considerable experimental as well as evaluation effort was expended in obtaining precise and consistent thermal constants. In the ENDF-6 format used to store the evaluated nuclear data these quantities are not given explicitly, they must be inferred from the resolved resonance parameters.

In the present work the resolved resonance region was parameterized within the multi-level Breit-Wigner formalism using the parameters based on the Atlas. Therefore, the thermal cross sections calculated from these parameters should reproduce the values recommended in the Atlas. Table 2 shows the thermal values for the total, elastic and capture cross sections, as well as the capture resonance integrals and these are compared with the Atlas. There are minor differences in the calculation of the thermal scattering cross sections which may be attributed to differences between the code applied in the present work and that used in the Atlas. The notable exception is the scattering cross section of ${ }^{145} \mathrm{Nd}$ which is due to differences in the parameters of the bound level in the Atlas as compared to that of Oh et al. [4], particularly the spin of the bound level. The other major difference between the present work and the Atlas value is the resonance capture integral of ${ }^{147} \mathrm{Nd}$ where the Atlas value, $430 \mathrm{~b}$, is computed for known resonances below $33 \mathrm{eV}$ while the present value, $530.9 \mathrm{~b}$, includes contributions from the unresolved resonance and fast neutron regions.

Fig. 1 illustrates some of our results in the thermal and resolved resonance regions by showing ${ }^{143,145} \mathrm{Nd}$ capture cross sections and comparing them to ENDF/B-VI.8, JEFF-3.1 and JENDL-3.3 libraries and to the measurements. It can be seen that our evaluations are in fairly good agreement with the data as well as with the other libraries.

\subsection{Unresolved resonances}

As a starting point, the average resolved resonance parameters were adopted from the Atlas. If average values were not recommended there, such as the $\mathrm{d}$-wave strength functions, the relevant quantities were derived from the systematics as represented in the figures shown in the Atlas. Adjustments in the average parameters were then carried out within their uncertainty limits until the calculations were consistent with the best measurements. In the present analysis the data of Wisshak et al. [36] below $213 \mathrm{keV}$ are judged to be the best. Above this energy, the measurements of [17] is considered as a guid- 
Table 2

Calculated thermal cross sections and capture resonance integrals for the Nd isotopes compared to values recommended in the Atlas of Neutron Resonances [11] (in barns).

\begin{tabular}{cccccc}
\hline Isotope & Reference & Total & Elastic & Capture & Resonance Integral \\
\hline${ }^{142} \mathrm{Nd}$ & present & 26.63 & 7.93 & 18.70 & 6.18 \\
& Atlas & $26.2 \pm 0.2$ & $7.5 \pm 0.5$ & $18.7 \pm 0.7$ & $34 \pm 11$ \\
${ }^{143} \mathrm{Nd}$ & present & 405.25 & 80.10 & 325.15 & 130.17 \\
& Atlas & $404.3 \pm 10.2$ & $79.3 \pm 2.0$ & $325 \pm 10$ & $129 \pm 30$ \\
${ }^{144} \mathrm{Nd}$ & present & 5.04 & 1.45 & 3.59 & 4.44 \\
& Atlas & $4.36 \pm 0.31$ & $1.0 \pm 0.2$ & $3.6 \pm 0.3$ & $4.2 \pm 0.5$ \\
${ }^{145} \mathrm{Nd}$ & present & 68.50 & 18.67 & 49.83 & 245.04 \\
& Atlas & $66.5 \pm 1.1$ & $16.5 \pm 0.4$ & $50.0 \pm 1.0$ & $230 \pm 35$ \\
${ }^{146} \mathrm{Nd}$ & present & 11.74 & 10.25 & 1.49 & 2.92 \\
& Atlas & $11.09 \pm 0.41$ & $9.6 \pm 0.4$ & $1.49 \pm 0.06$ & $2.57 \pm 0.14$ \\
${ }^{147} \mathrm{Nd}$ & present & 551.66 & 111.09 & 440.57 & 539.83 \\
& Atlas & - & - & $440 \pm 150$ & 430 \\
\multirow{2}{*}{${ }^{148} \mathrm{Nd}$} & present & 6.96 & 4.38 & 2.58 & 15.94 \\
& Atlas & $6.92 \pm 0.41$ & $4.34 \pm 0.5$ & $2.58 \pm 0.07$ & $15.5 \pm 1.5$ \\
$150 \mathrm{Nd}$ & present & 5.66 & 4.62 & 1.04 & 15.57 \\
& Atlas & $5.64 \pm 0.40$ & $4.6 \pm 0.4$ & $1.04 \pm 0.04$ & $15.2 \pm 0.8$ \\
\hline
\end{tabular}
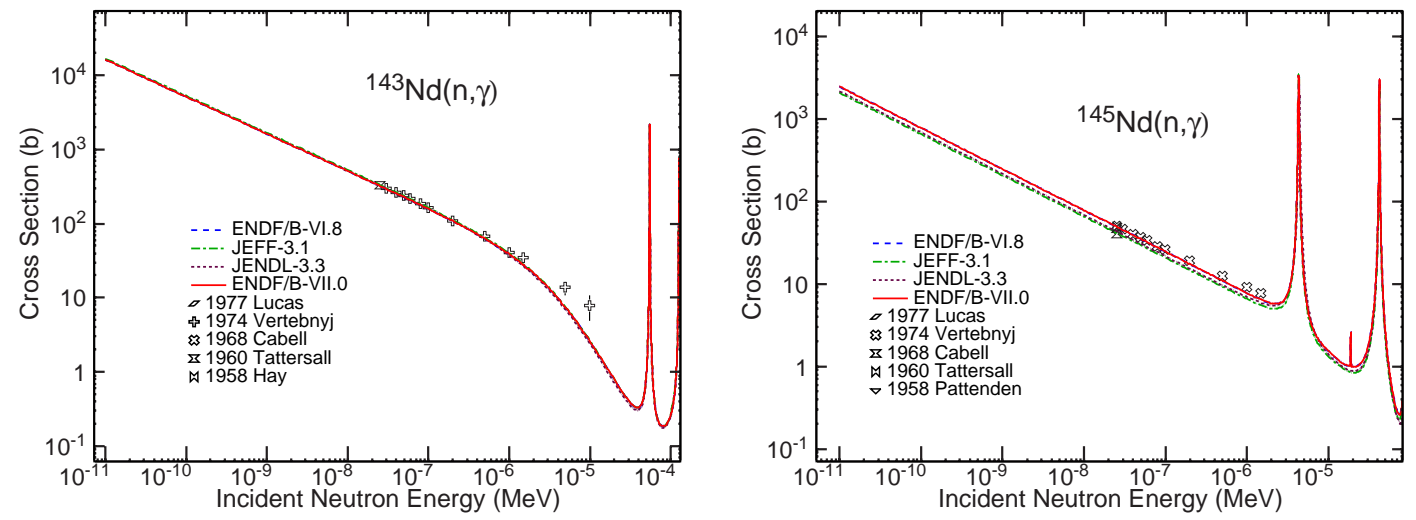

Fig. 1. Evaluated capture cross sections for ${ }^{143,145} \mathrm{Nd}$ in the thermal and resolved resonance region compared to other evaluated libraries and the measurements [30-35]. Our results are shown in red. 
ance in the fitting procedure. For ${ }^{150} \mathrm{Nd}$, only one cross cross data set in the unresolved energy region is available [37], while for radioactive ${ }^{147} \mathrm{Nd}$ no experimental data are reported. The final evaluated values are given in Table 3 and are compared with the recommendations of Mughabghab as given in the Atlas.

Table 3

Unresolved neutron resonance parameters for $\mathrm{Nd}$ isotopes. The present results are compared with the Atlas for the average resonance width, $\langle D\rangle$, the average capture width, $\left\langle\Gamma_{\gamma}\right\rangle$, and the neutron strength function, $\mathrm{S}_{n}$.

\begin{tabular}{ccc|ccc|ccc}
\hline Isotope & Source & $<D>, \mathrm{eV}$ & \multicolumn{3}{|c|}{$<\Gamma_{\gamma}>, \mathrm{meV}$} & \multicolumn{3}{c}{$\mathrm{S}_{n}, \mathrm{x}^{-4}$} \\
& & s-wave & s-wave & p-wave & d-wave & s-wave & p-wave & d-wave \\
\hline${ }^{142} \mathrm{Nd}$ & present & 1170 & 48.5 & 43.5 & 46 & 1.7 & 0.58 & 1.45 \\
& Atlas & $1035 \pm 135$ & $67 \pm 8$ & $48 \pm 5$ & - & $1.45 \pm 0.49$ & $0.58 \pm 0.13$ & - \\
\multirow{2}{*}{${ }^{143} \mathrm{Nd}$} & present & 36.0 & 79.9 & 40 & 79.9 & 3.62 & 0.8 & 1.5 \\
& Atlas & $37.6 \pm 2.1$ & $73 \pm 4$ & - & - & $3.67 \pm 0.55$ & $0.85 \pm 0.31$ & - \\
${ }^{144} \mathrm{Nd}$ & present & 450 & 57.0 & 43 & 48 & 5.36 & 0.92 & 3.0 \\
& Atlas & $450 \pm 50$ & $51 \pm 4$ & $36 \pm 6$ & - & $5.36 \pm 1.16$ & $0.77 \pm 0.15$ & - \\
${ }^{145} \mathrm{Nd}$ & present & 18.0 & 76.5 & 40 & 76.5 & 4.75 & 1.2 & 0.8 \\
& Atlas & $17.8 \pm 0.7$ & $58 \pm 5$ & - & - & $4.46 \pm 0.44$ & $0.8 \pm 0.4$ & - \\
\multirow{2}{*}{${ }^{146} \mathrm{Nd}$} & present & 210 & 44.0 & 30 & 36 & 3.0 & 1.6 & 1.25 \\
& Atlas & $235 \pm 29$ & $54 \pm 6$ & 23 & - & $2.6 \pm 0.4$ & $1.2 \pm 0.4$ & - \\
\multirow{2}{*}{${ }^{147} \mathrm{Nd}$} & present & 6.3 & 68.8 & 29 & 45 & 3.4 & 1.2 & 3.0 \\
& Atlas & $4.4 \pm 1.5$ & 75.0 & - & - & - & - & - \\
${ }^{148} \mathrm{Nd}$ & present & 165 & 46.0 & 34 & 40 & 3.96 & 1.09 & 3.0 \\
& Atlas & $179 \pm 14$ & $39 \pm 5$ & - & - & $3.29 \pm 0.67$ & $0.92 \pm 0.17$ & - \\
${ }^{150} \mathrm{Nd}$ & present & 169 & 61.0 & 41 & 50 & 3.4 & 1.2 & 3.0 \\
& Atlas & $169 \pm 11$ & $67 \pm 25$ & - & - & $3.0 \pm 0.4$ & $0.8 \pm 0.2$ & - \\
\hline
\end{tabular}

Upper energies of the unresolved resonance regions are usually set equal to the energy of the first excited level of the target nucleus. However, the first excited levels in ${ }^{142,144} \mathrm{Nd}$ are relatively high, which makes it difficult to find a smooth match between the unresolved resonance region and the fast neutron region. Accordingly, in the two above mentioned cases, the upper boundaries of the unresolved resonance regions were lowered to ensure smooth match with the fast neutron region. The quality of our evaluations along with these mergings is illustrated in Fig. 2 for capture cross sections on all considered neodymium isotopes. The adopted boundaries of the unresolved resonance regions are listed in Table 1. 

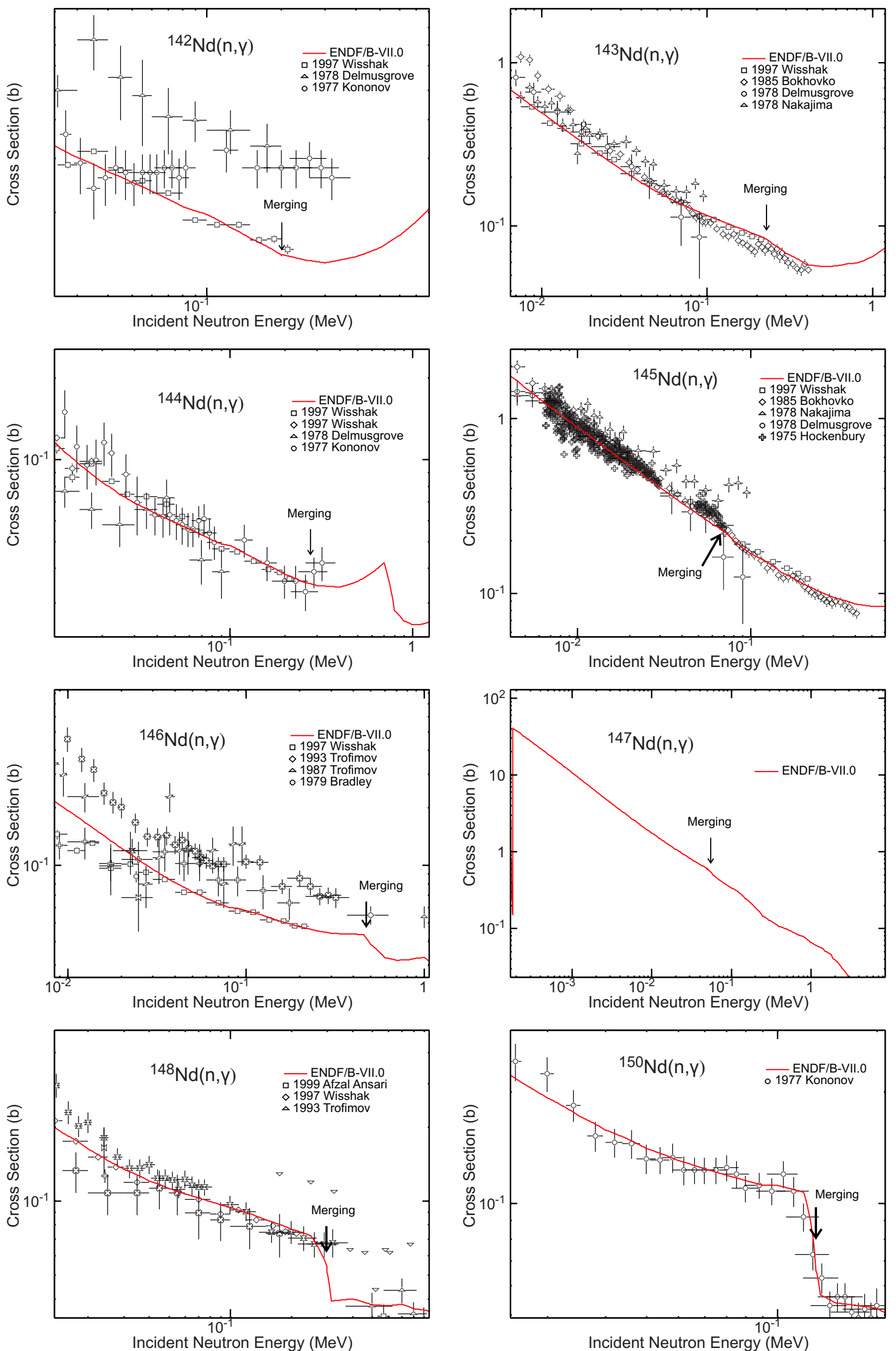

Fig. 2. Evaluated capture cross sections for all Nd isotopes in the unresolved resonance region compared to the measurements $[16,38,39,17,40-42,20]$. Our evaluations are shown in red. 


\section{Simultaneous evaluation in the fast neutron region}

The simultaneous evaluation of a complete isotopic chain is an important aspect of our procedure. As described in Sec. 3, the combined capabilities of the Atlas of Neutron Resonances, the nuclear reaction model code EMPIRE, input parameter libraries such as the Reference Input Parameter Library (RIPL-2), experimental cross section library CSISRS/EXFOR, and numerous ENDF formatting and checking utilities have expedited evaluation procedures and made newly evaluated files more complete and reliable. The modern nuclear reaction codes are sufficiently powerful and flexible so that evaluators can search for a coherent set of parameters that defines recommended cross sections for all members of the isotopic chain.

We take advantage of the fact that certain critical model parameters, such as optical potential and asymptotic value of the level density parameter, vary smoothly as function of mass number. Accordingly, they do not differ too much among individual members of the isotopic family. Taking into account known mass dependencies or trends for certain model parameters we were able to use experimental data for the neighboring isotopes and for the natural element to constrain model parameters for individual isotopes even if no measurements on a given isotope/reaction are available. When doing this, we accounted for the prominent, and well understood, differences between various isotopes such as binding energies, deformations, and discrete level schemes. Adequate consideration of these factors is critical for the reliable implementation of the procedure. Fortunately, most of these quantities are known with a reasonable level of confidence. This justifies ascribing some residual discrepancies between calculations and experiments to the less known but slowly varying parameters that can be adjusted by tuning the related systematics. Implementation of such consistent set in reaction calculations ensures that the differences among evaluations can be understood in terms of the physical differences among the isotopes (e.g., different ground state deformations).

\subsection{Determination of the optical model potential (OMP)}

The total cross section is best known for ${ }^{144} \mathrm{Nd}$. There are measurements by Wisshak et al. [16] in the unresolved resonance region and by Shamu et al. [43] from $0.7 \mathrm{MeV}$ up to $14 \mathrm{MeV}$, and a single-energy point by Djumin et al. [44] at $14.2 \mathrm{MeV}$. In addition, there is the measurement of the elastic angular distribution at $7.0 \mathrm{MeV}$ by Haouat et al. [45]. These data allow to derive parameters of the optical model potential within acceptable margins. Our results are discussed below, shown later are Figs. 8 and 10 confirming that calculated total cross sections reproduce the reported experimental data [43- 
$45]$.

The situation for other neodymium isotopes, ${ }^{142,143,145,146,148} \mathrm{Nd}$, is not so favorable. There exist measurements by Wisshak et al. [16] in the unresolved resonance region, and single energy-points at $14.2 \mathrm{MeV}$ by Djumin et al. [44] (the latter is not available for the radioactive ${ }^{147} \mathrm{Nd}$ ) but these data do not constrain model calculations sufficiently to determine optical model parameters. To overcome this difficulty we resorted to the wealth of measurements $[15,46-$ 50] on natural neodymium. Although these data do not allow to address individual isotopes, they provide an overall energy dependence that can be refined by referring to isotopic measurements. For instance, the elastic angular distributions measured by Haouat et al. [45] can be used to adjust OMP to describe interaction of neutrons with ${ }^{142,146,148} \mathrm{Nd}$ isotopes.

We used spherical optical models to calculate transmission coefficients for all ejectiles involved in the reactions. However, the incident channel was treated in terms of the coupled-channels formalism rather than the spherical optical model. Accordingly, a proper coupling between collective levels was essential for describing cross sections and angular distributions of neutrons inelastically scattered to collective levels. In certain cases we also included direct scattering to the collective levels embedded in the continuum. We performed coupledchannels calculations for the ground state rotational bands in ${ }^{143,145,147} \mathrm{Nd}$ as shown in Table 4 . For ${ }^{142,144} \mathrm{Nd}$, the harmonic vibrational coupled-channels calculations were employed for the low-lying one-phonon and two-phonon discrete levels. For the ${ }^{146,148,150} \mathrm{Nd}$ isotopes we included DWBA calculations to a number of collective levels in addition to the standard coupled-channels treatment of the ground state rotational band. We note, that in the latter three isotopes we allowed for discrete levels embedded in the continuum to improve high energy parts of neutron spectra.

The initial optical potential parameters for neutrons were based on the spherical parameterizations developed by Koning et al. [51]. Since the neodymium isotopes are deformed and require the use of the DWBA and CC formalisms proper adjustments of the spherical potentials were needed for physically meaningful description of all reaction channels. These adjustments were performed retaining flexible functional form of the original Koning's parameterization. The real volume part of the potential is represented as

$$
V_{V}(E)=v_{1}\left[1-v_{2}\left(E-E_{f}\right)+v_{3}\left(E-E_{f}\right)^{2}-v_{4}\left(E-E_{f}\right)^{3}\right],
$$

the imaginary volume part of the potential reads

$$
W_{V}(E)=w_{1} \frac{\left(E-E_{f}\right)^{2}}{\left(E-E_{f}\right)^{2}+w_{2}^{2}}
$$


Table 4

The ground state rotational band and its quadrupole deformation parameter $\left(\beta_{2}\right)$ used in the coupled-channels calculations for ${ }^{143,145,147} \mathrm{Nd}$.

\begin{tabular}{cccc}
\hline Isotope & $\mathrm{E}, \mathrm{MeV}$ & $J^{\pi}$ & $\beta_{2}$ \\
\hline${ }^{143} \mathrm{Nd}$ & 0.0000 & $7 / 2^{-}$ & 0.100 \\
& 1.4071 & $9 / 2^{-}$ & $\prime \prime$ \\
& 1.4312 & $11 / 2^{-}$ & $\prime \prime$ \\
${ }^{145} \mathrm{Nd}$ & 0.0000 & $7 / 2^{-}$ & 0.114 \\
& 0.6577 & $11 / 2^{-}$ & $\prime \prime$ \\
& 0.7483 & $9 / 2^{-}$ & $\prime \prime$ \\
${ }^{147} \mathrm{Nd}$ & 0.0000 & $5 / 2^{-}$ & 0.151 \\
& 0.0499 & $7 / 2^{-}$ & $\prime \prime$ \\
& 0.1903 & $9 / 2^{-}$ & $\prime \prime$ \\
\hline
\end{tabular}

the surface imaginary potential is expressed by

$$
W_{D}(E)=d_{1} \frac{\left(E-E_{f}\right)^{2}}{\left(E-E_{f}\right)^{2}+d_{3}^{2}} \exp \left[-d_{2}\left(E-E_{f}\right)\right],
$$

the real spin-orbit term is

$$
V_{S O}(E)=v_{s o 1} \exp \left[-v_{s o 2}\left(E-E_{f}\right)\right]
$$

and its imaginary counterpart is

$$
W_{S O}(E)=w_{s o 1} \frac{\left(E-E_{f}\right)^{2}}{\left(E-E_{f}\right)^{2}+\left(w_{s o 2}\right)^{2}},
$$

where $v_{i}, d_{i}$ and $w_{i}$ are the parameters and $E_{f}$ is the Fermi energy. The form factor is of the usual Woods-Saxon shape,

$$
f\left(r, R_{i}, a_{i}\right)=\left(1+\exp \left[\left(r-R_{i}\right) / a_{i}\right]\right)^{-1}
$$

and the nuclear radius is given as

$$
R_{i}=r_{i} A^{1 / 3}
$$

where $A$ is the atomic mass number, and the geometrical parameters, $r_{i}$ and $a_{i}$, stand for the radius and diffuseness, respectively. In all cases, these geometrical parameters were assumed to be energy-independent. The neutron optical model parameters used in the present work are given in Table 5.

For protons we consistently used parameterization by Koning et al. [51] and for $\alpha$-particles the parameterization by Avrigeanu et al. [52]. Since emission 
of both charge particles from neodymium isotopes is of limited importance for applications, we adopted these global potentials without any modifications. This choice is also justified by the overwhelming contribution of the preequilibrium processes to the $(\mathrm{n}, \mathrm{p})$ and $(\mathrm{n}, \alpha)$ reactions on the neodymium isotopes.

Table 5

Neutron coupled-channels optical model parameters used in the evaluations of $\mathrm{Nd}$ isotopes. For explanation of parameters see Eqs.(1-7), $r_{i}$ and $a_{i}$ are given in fm, $v_{i}, d_{i}$ and $w_{i}$ in $\mathrm{MeV}, \mathrm{MeV}^{-1}$ or $\mathrm{MeV}^{-2}$ as applicable, and $\mathrm{E}_{f}$ in $\mathrm{MeV}$. The coupling schemes are discussed in the text.

\begin{tabular}{|c|c|c|c|c|c|c|c|c|}
\hline & ${ }^{142} \mathrm{Nd}$ & ${ }^{143} \mathrm{Nd}$ & ${ }^{144} \mathrm{Nd}$ & ${ }^{145} \mathrm{Nd}$ & ${ }^{146} \mathrm{Nd}$ & ${ }^{147} \mathrm{Nd}$ & ${ }^{148} \mathrm{Nd}$ & ${ }^{150} \mathrm{Nd}$ \\
\hline $\mathrm{v}_{1}$ & 50.2 & 51.2 & 52.6 & 51.6 & 51.658 & 51.6 & 51.658 & 51.8 \\
\hline $\mathrm{v}_{2}$ & 0.0075 & 0.0085 & 0.0089 & 0.0085 & 0.007 & 0.0085 & 0.0073 & 0.0073 \\
\hline $\mathrm{v}_{3}$ & $1.7 \times 10^{-5}$ & $1.7 \times 10^{-5}$ & $1.7 \times 10^{-5}$ & $1.7 \times 10^{-5}$ & $1.7 \times 10^{-5}$ & $1.7 \times 10^{-5}$ & $1.7 \times 10^{-5}$ & $1.7 \times 10^{-5}$ \\
\hline $\mathrm{v}_{4}$ & $7.0 \times 10^{-9}$ & $7.0 \times 10^{-9}$ & $7.0 \times 10^{-9}$ & $7.0 \times 10^{-9}$ & $7.0 \times 10^{-9}$ & $7.0 \times 10^{-9}$ & $7.0 \times 10^{-9}$ & $7.0 \times 10^{-9}$ \\
\hline$r_{V}$ & 1.253 & 1.247 & 1.252 & 1.247 & 1.25 & 1.247 & 1.255 & 1.255 \\
\hline$a_{V}$ & 0.66 & 0.66 & 0.64 & 0.66 & 0.64 & 0.66 & 0.67 & 0.68 \\
\hline$w_{1}$ & 14.2 & 14.2 & 14.2 & 14.2 & 14.2 & 14.2 & 14.2 & 14.2 \\
\hline$w_{2}$ & 86 & 86 & 86 & 86 & 86 & 86 & 86 & 86 \\
\hline$r_{D}$ & 1.25 & 1.258 & 1.258 & 1.258 & 1.258 & 1.258 & 1.258 & 1.258 \\
\hline$a_{D}$ & 0.55 & 0.58 & 0.56 & 0.58 & 0.58 & 0.58 & 0.55 & 0.54 \\
\hline$d_{1}$ & 13.0 & 14.0 & 12.8 & 14.0 & 14.0 & 14.0 & 14.6 & 14.0 \\
\hline$d_{2}$ & 0.0276 & 0.0276 & 0.276 & 0.276 & 0.276 & 0.276 & 0.276 & 0.276 \\
\hline$d_{3}$ & 13.2 & 13.2 & 13.2 & 13.2 & 13.2 & 13.2 & 13.2 & 13.2 \\
\hline$r_{S O}$ & 1.08 & 1.08 & 1.08 & 1.08 & 1.08 & 1.08 & 1.08 & 1.08 \\
\hline$a_{S O}$ & 0.59 & 0.59 & 0.59 & 0.59 & 0.59 & 0.59 & 0.59 & 0.59 \\
\hline $\mathrm{v}_{s o 1}$ & 6.4 & 6.4 & 6.4 & 6.4 & 6.4 & 6.4 & 6.4 & 6.4 \\
\hline $\mathrm{v}_{s o 2}$ & 0.004 & 0.004 & 0.004 & 0.004 & 0.004 & 0.004 & 0.004 & 0.004 \\
\hline $\mathrm{W}_{s o 1}$ & -3.1 & -3.1 & -3.1 & -3.1 & -3.1 & -3.1 & -3.1 & -3.1 \\
\hline $\mathrm{w}_{s o 2}$ & 160 & 160 & 160 & 160 & 160 & 160 & 160 & 160 \\
\hline$E_{f}$ & -7.97 & -7.97 & -7.97 & -7.97 & -7.97 & -7.97 & -7.97 & -7.97 \\
\hline
\end{tabular}

\subsection{Comparison of evaluations for various isotopes}

Our parameterization of the optical model was validated by comparing calculated total cross sections and elastic scattering angular distributions with the 
experimental data in the unresolved resonance and fast neutron regions.

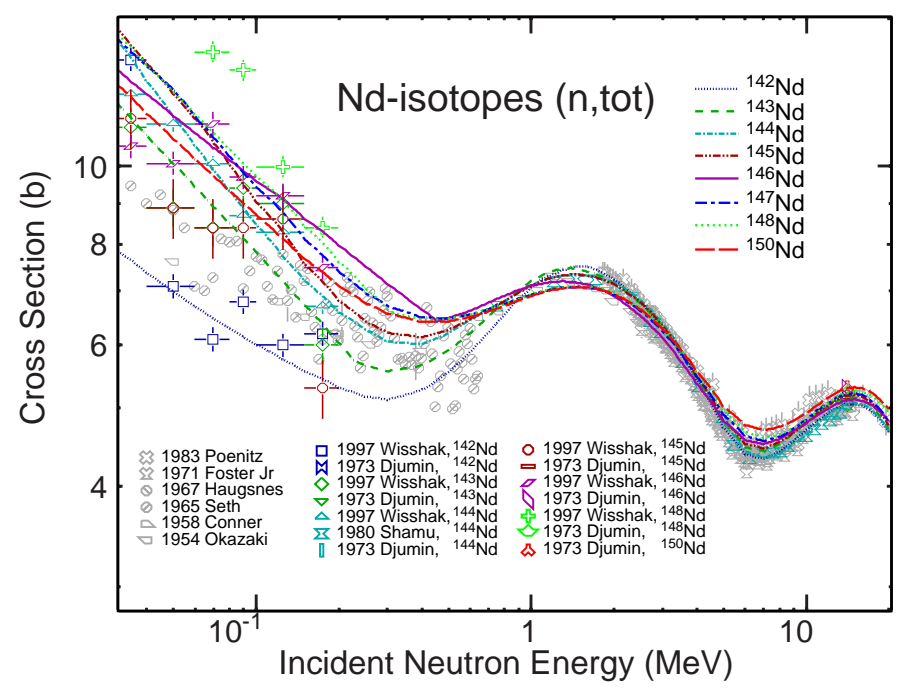

Fig. 3. Total cross sections for $\mathrm{Nd}$ isotopes calculated using coupled-channels formalism with optical potentials of Table 5 compared to the experimental results $[15,46-50,16,44,43]$.

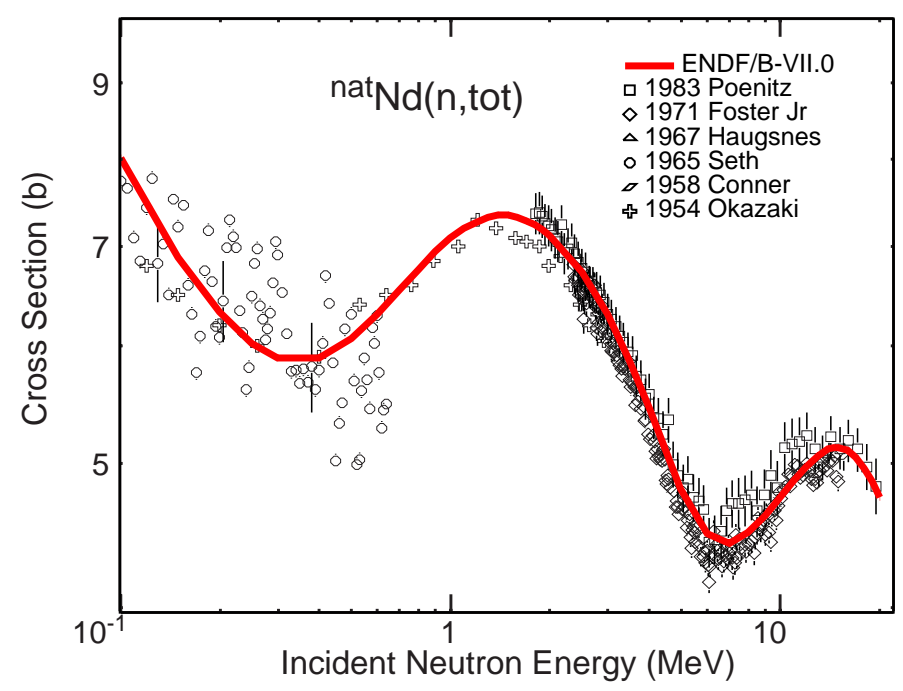

Fig. 4. Calculated total cross sections for ${ }^{n a t} \mathrm{Nd}$ compared with the measurements $[15,46-50]$.

Fig. 3 summarizes results for the total cross sections by comparing our isotopic calculations with experimental data taken on ${ }^{n a t} \mathrm{Nd}[15,46-50]$ as well as on the individual isotopes $[16,43,44]$. Our coupled-channels calculations are divergent below $1 \mathrm{MeV}$ and tend to approach each other above. In the latter range they closely follow measurements on ${ }^{n a t} \mathrm{Nd}$. In the lower energy range the calculations grasp the main tendencies revealed by the experimental data. Fig. 4 demonstrates how well the calculated total cross sections on ${ }^{\text {nat }}$ Nd reproduce measurements . For the purpose of this comparison, the calculated cross 
sections for ${ }^{\text {nat }} \mathrm{Nd}$ were obtained as a sum of cross sections for all individual isotopes weighted by their respective abundances.

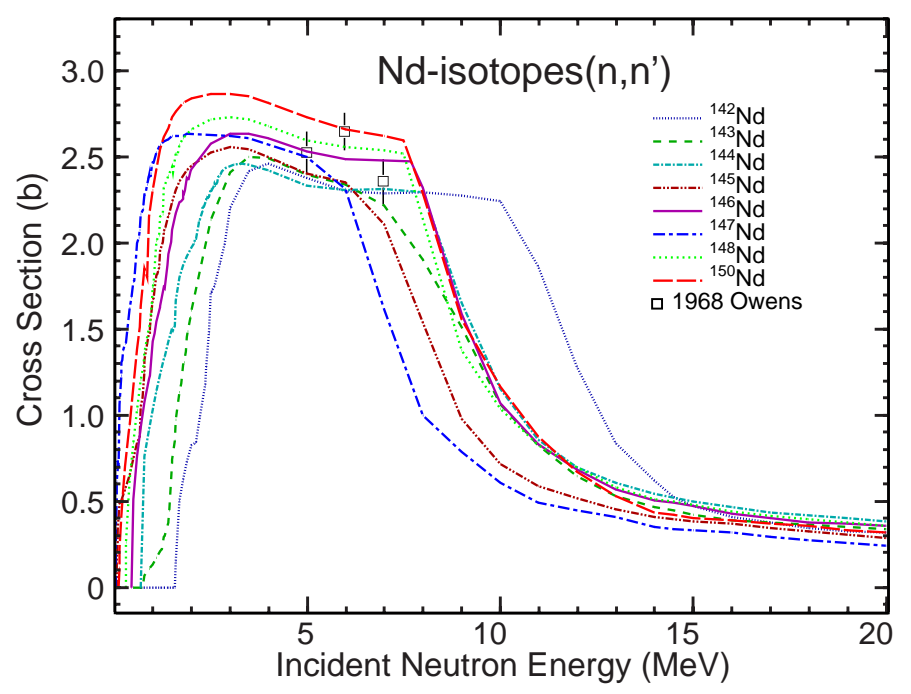

Fig. 5. Inelastic cross sections for all Nd isotopes compared to the measurement on ${ }^{n a t} \mathrm{Nd}[53]$.

The agreement is also good for the elastic scattering angular distributions for $142,144,146,148 \mathrm{Nd}$ at $7.0 \mathrm{MeV}$ [45] as shown later in Fig. 10. We note, that these angular distributions were included in the fit to constrain optical potential on different isotopes.

We illustrate advantages of the simultaneous evaluation by plotting together evaluated cross sections for the major reaction channels for all isotopes of neodymium. We anticipate that our results, in addition to the optical model potential, depend also on other parameters involved in the calculations. Fig. 5 shows the inelastic cross sections compared to the experimental data on ${ }^{n a t} \mathrm{Nd}$ [53]. This relatively old (1968) experiment does not provide a stringent test of the calculations but the measurements are consistent with the new evaluations. We note that our $\left(\mathrm{n}, \mathrm{n}^{\prime}\right)$ cross sections near $20 \mathrm{MeV}$ are in the range of 300-400 $\mathrm{mb}$ due to the strong contribution of the preequilibrium and direct reaction processes.

Fig. 6 shows $(\mathrm{n}, 2 \mathrm{n})$ cross sections for a series of neodymium isotopes. The results obtained for $142,144,146,148,150 \mathrm{Nd}$ reproduce the available experimental data. In the case of the remaining isotopes, ${ }^{143,145,147} \mathrm{Nd}$, for which there are no measurements, we obtain consistent shapes and trends due to the homogeneous modeling involved in the evaluation procedure.

Fig. 7 shows capture cross sections compared to the selected sets of experimental data obtained after elimination of raw and apparently wrong results. One can see that we consistently reproduce the data for all isotopes. 


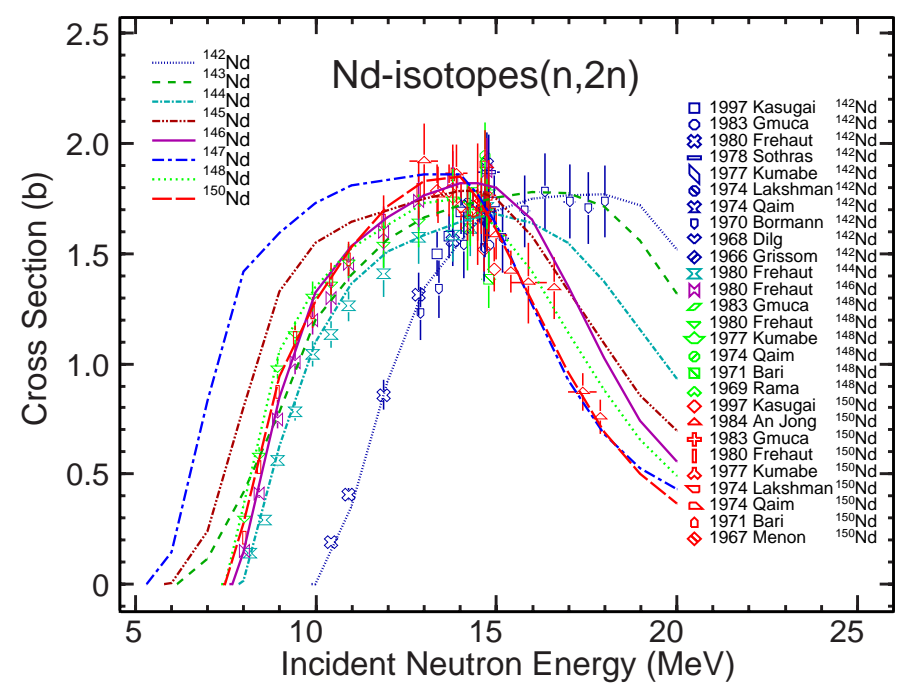

Fig. 6. The $(\mathrm{n}, 2 \mathrm{n})$ cross sections for all Nd isotopes compared with the measurements $[21,22,54-62,23,63,64]$.

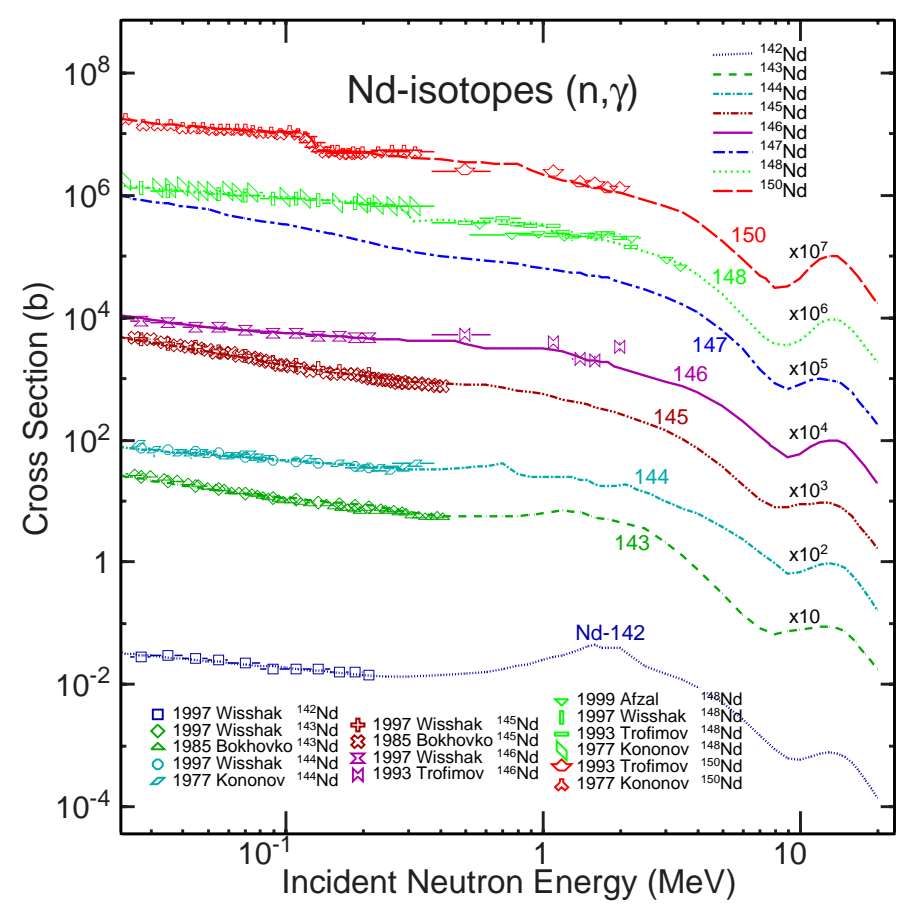

Fig. 7. Capture cross sections for the Nd isotopes compared with the measurements $[16,17,41,19,20]$.

\subsection{Discussion of individual reactions}

In general, we restrict our discussion to those Nd isotopes where experimental data are available. Also, we consider practical importance of cross sections and focus on the two priority isotopes, ${ }^{143,145} \mathrm{Nd}$. 

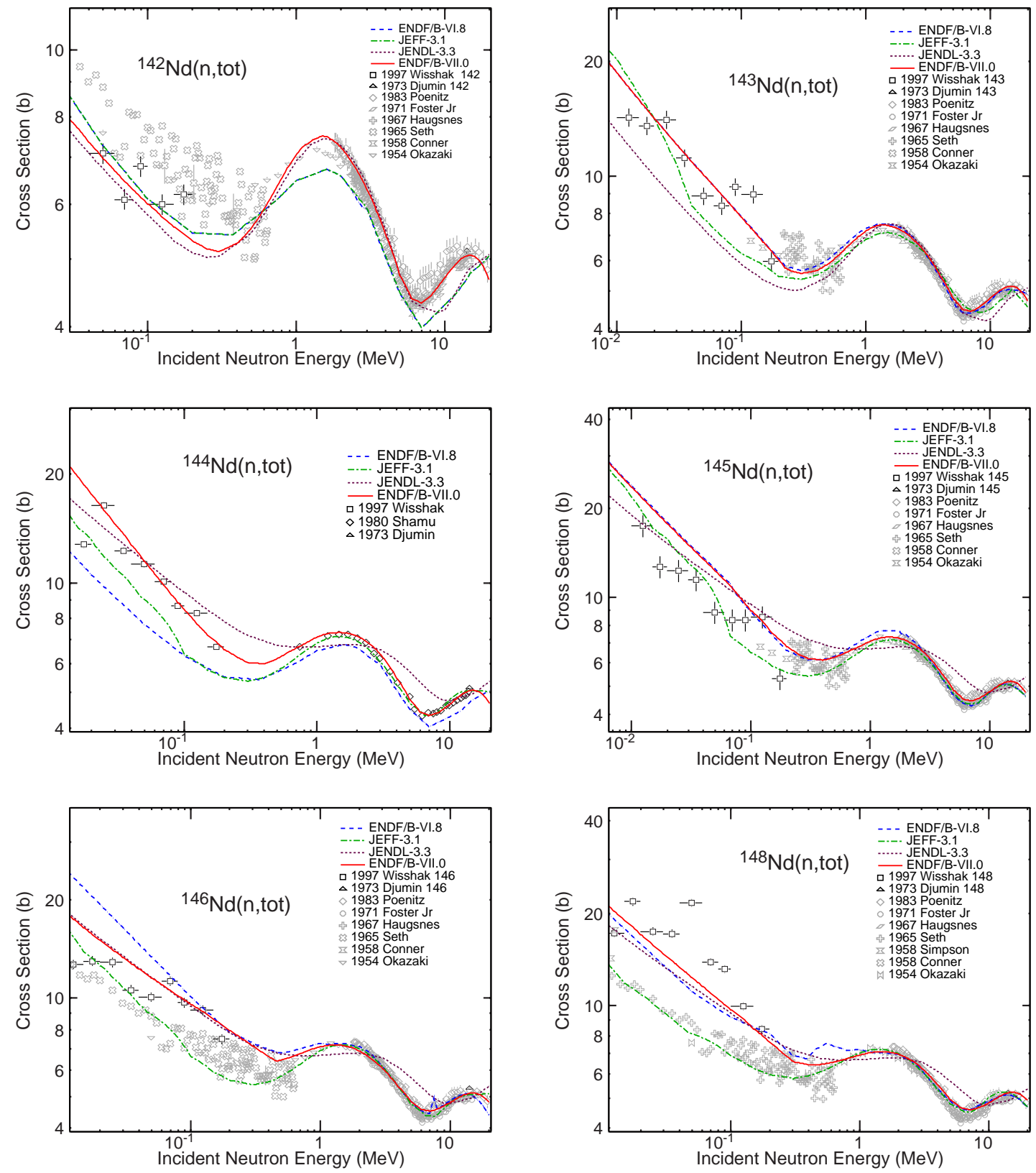

Fig. 8. Evaluated total cross sections for all Nd isotopes in the fast neutron energy region compared to other evaluations and to the isotopic measurements $[16,43,44]$. Shown for comparison in lighter color are measured data on ${ }^{\text {nat }} \mathrm{Nd}[15,46-50]$.

\subsubsection{Total cross sections}

Fig. 8 shows total cross sections for the $\mathrm{Nd}$ isotopes where experimental data are available. Our results are compared with the ENDF/B-VI.8, JEFF-3.1 and JENDL-3.3 evaluations. The data measured by Wisshak et al. [16] were used as a guide for the evaluation of ${ }^{142-146,148} \mathrm{Nd}$ in the unresolved resonance region. In order to ensure smooth cross sections, we disregarded several measurements which deviated too much, see ${ }^{145,148} \mathrm{Nd}$. In the fast neutron region, 

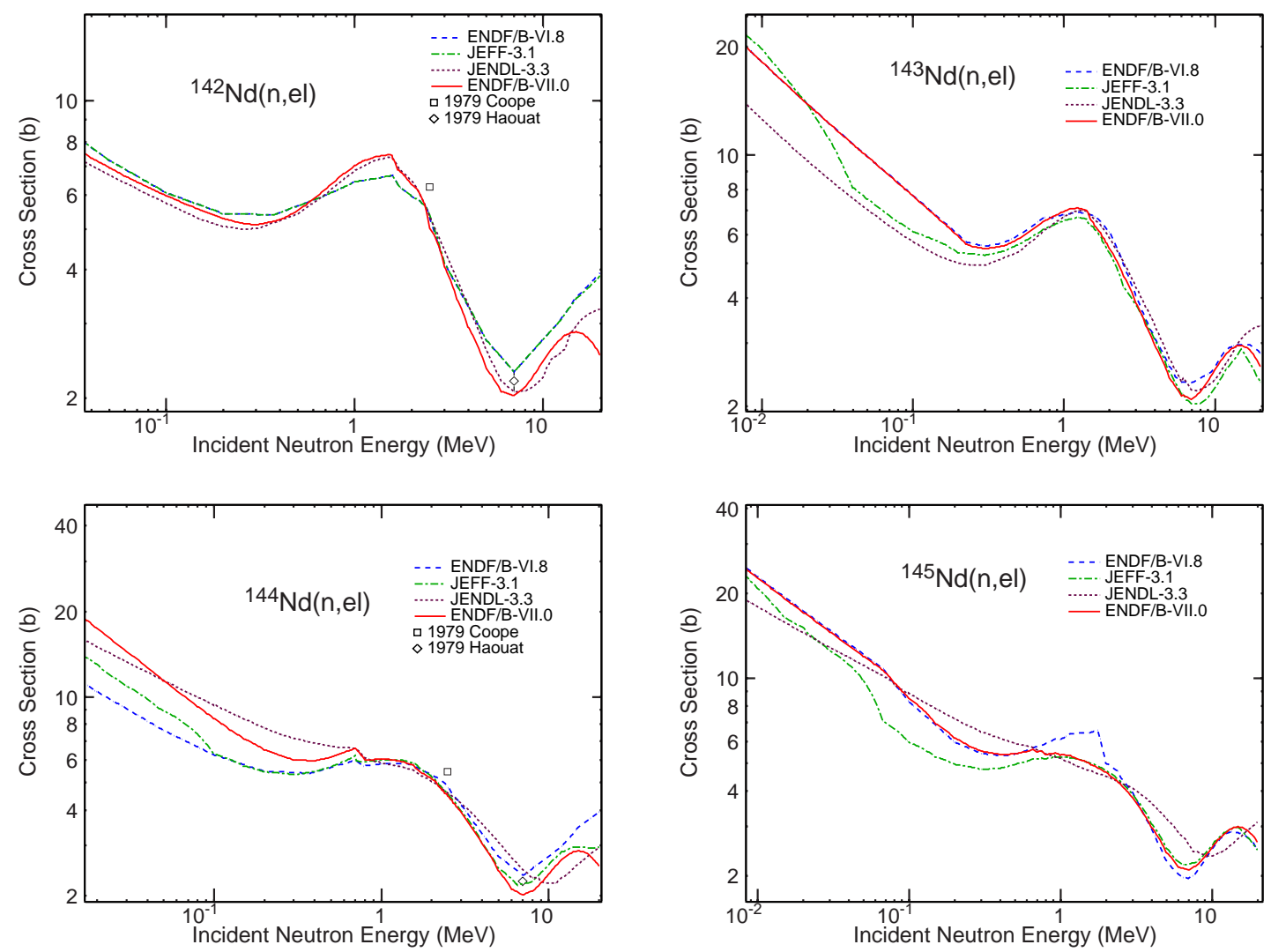

Fig. 9. Evaluated elastic cross sections for ${ }^{142-145} \mathrm{Nd}$ in the fast neutron region compared to other evaluations and to the measurements $[65,45]$.

our evaluations normally considered the experimental data on the natural neodymium $[46,49,48,50,15,47]$, because there is not sufficient experimental information available for the individual isotopes. Actually, in most cases there exist only one point measured by Djumin et al. at $14.2 \mathrm{MeV}$. The only exception is ${ }^{144} \mathrm{Nd}$ for which total cross sections were measured by Shamu et al. [43] from $0.7 \mathrm{MeV}$ to $14 \mathrm{MeV}$.

We started our evaluation with adjusting the optical model parameters to reproduce total cross sections [43] and elastic angular distributions [45] on ${ }^{144} \mathrm{Nd}$. The resulting optical model parameters for ${ }^{144} \mathrm{Nd}$ were subsequently used as an initial guess for the remaining neodymium isotopes. They were adjusted to reproduce measured data for the total cross sections on ${ }^{\text {nat }} \mathrm{Nd}[46,49,48,50,15,47]$ and isotopic Nd [44], and angular distributions of the elastic scattering on isotopic Nd [45]. Our evaluations reproduce the isotopic experimental data well as shown in Fig. 8. Although the total cross section of ${ }^{146} \mathrm{Nd}$ differs by $3.3 \%$ from the value measured by Djumin et al. [44] at $14.2 \mathrm{MeV}$, we retain the agreement to be acceptable in view of the experimental uncertainty. The total cross sections for the radioactive isotope ${ }^{147} \mathrm{Nd}$, for which no experimental data are available, were computed using the optical model parameters deduced for ${ }^{145} \mathrm{Nd}$. 

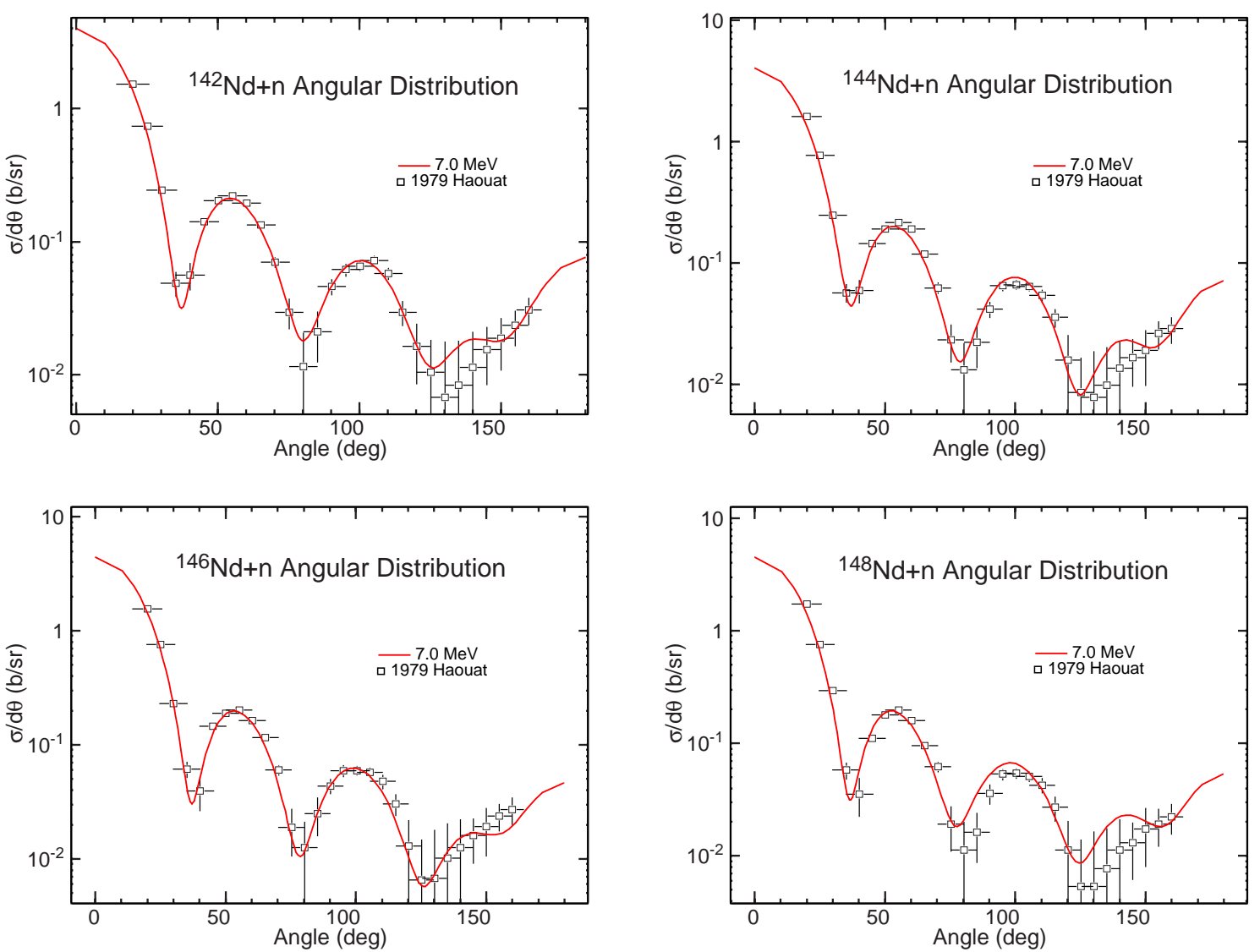

Fig. 10. Evaluated neutron elastic scattering angular distributions for $142,144,146,148 \mathrm{Nd}$ at $\mathrm{E}_{n}=7.0 \mathrm{MeV}$ compared with the isotopic measurements of Haouat et al. [45].

\subsubsection{Neutron elastic cross sections}

The angle-integrated elastic cross sections were reconstructed by subtracting non-elastic cross sections from the total cross sections. Fig. 9 illustrates our results by showing elastic cross sections on ${ }^{142-145} \mathrm{Nd}$ and comparing them to the ENDF/B-VI.8, JEFF-3.1 and JENDL-3.3 evaluations and to the measured values $[65,45]$. For ${ }^{142,144} \mathrm{Nd}$ we reproduce, within the uncertainties, the experimental data at 7.0 MeV obtained by Haouat et al. [45], but we somewhat underestimate cross sections measured at $2.5 \mathrm{MeV}$ by Coope [65]. These results look reasonable and give some confidence in the ${ }^{143,145} \mathrm{Nd}$ evaluations where no experimental data are available.

The measured angular distributions at $7.0 \mathrm{MeV}$ [45] are well reproduced, see Fig. 10. This is not surprising as these data were used to constrain optical model parameters. Slight deviations observed at backward angles raise no serious concerns since the cross sections are very low. Although there is no experimental evidence in favor of the fourth bump seen in the calculated angular distributions the absolute values agree within the uncertainties. 

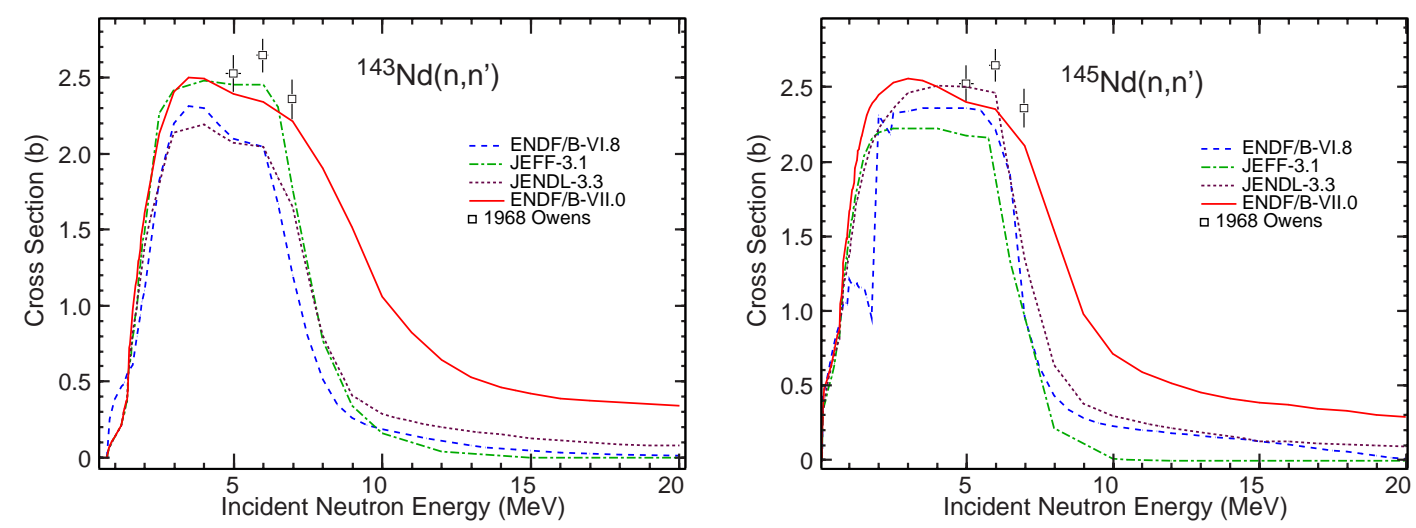

Fig. 11. Evaluated inelastic cross sections for ${ }^{143,145} \mathrm{Nd}$ compared to other evaluations and to the measurement by Owens et al. on ${ }^{n a t} \mathrm{Nd}$ [53].
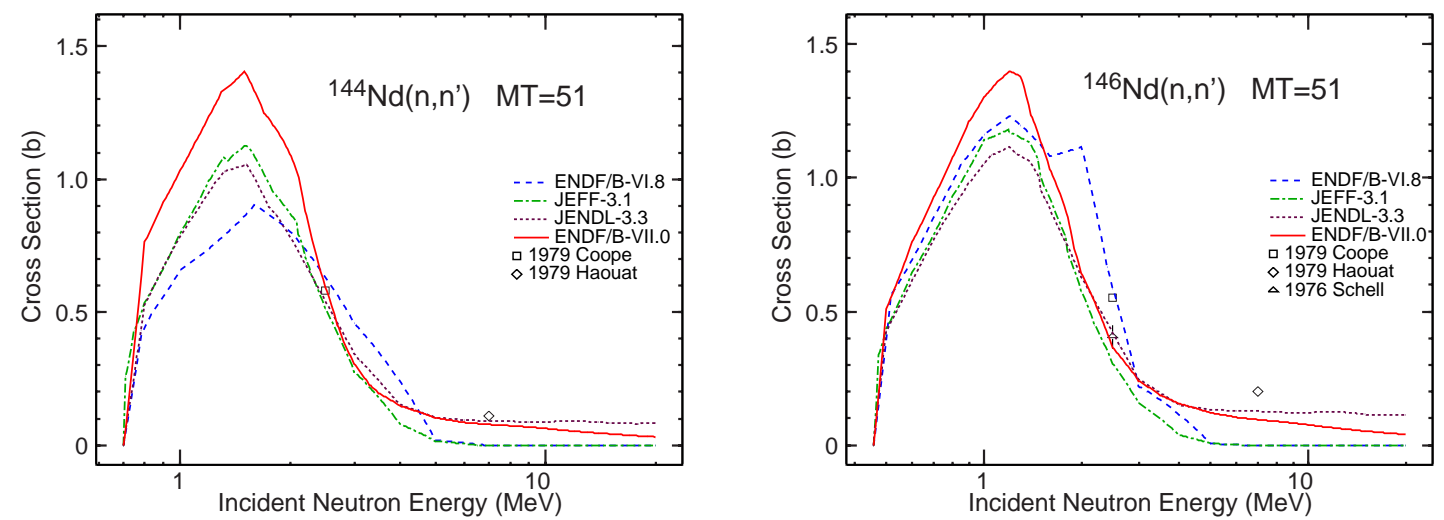

Fig. 12. Partial inelastic cross sections to the first excited level $(\mathrm{MT}=51$ in the ENDF-6 format notation) in ${ }^{144,146} \mathrm{Nd}$ compared with other evaluations and with the measurements $[65,45,66]$.

\subsubsection{Neutron inelastic cross sections}

Fig. 11 illustrates present evaluations of inelastic cross sections and compares ${ }^{143,145} \mathrm{Nd}(\mathrm{n}, \mathrm{n}$ ') to other libraries and to the measurement by Owens et al. [53]. We note, that the latter data are for ${ }^{n a t} \mathrm{Nd}$ since no measurements of total inelastic scattering are available for any of the neodymium isotopes. The comparison supports our calculations in the region of a plateau starting about $2 \mathrm{MeV}$ above the threshold. The cross sections within the plateau are about $2500 \mathrm{mb}$ for all the isotopes, which is consistent with the data of Owens et al.

Due to the use of the coupled-channels (CC) and multi-step direct (MSD) mechanisms in the modeling, our inelastic cross sections around $20 \mathrm{MeV}$ approach 300 - $400 \mathrm{mb}$. Earlier evaluations neglected these processes and their predictions are considerably lower. In some other cases, such as ${ }^{142,144,147} \mathrm{Nd}$ in ENDF/B-VI.8, nonphysical behavior cross sections at the highest energies is due to the apparent neglect of the $(n, 2 n)$ competition. 
There are several measurements of inelastic scattering to the first excited levels in ${ }^{142,144,146,148} \mathrm{Nd}$. Fig. 12 illustrates our results and compares ${ }^{144,146} \mathrm{Nd}\left(\mathrm{n}, \mathrm{n}_{1}{ }^{\prime}\right)$ cross sections with the measurement by Haouat et al. [45]. The figure indicates that the present evaluation, along with JENDL-3.3, provides better description of the experimental data than the other two evaluations.

\subsubsection{Neutron capture cross sections}

There is a relatively large amount of experimental data for neutron capture on all neodymium isotopes, except for ${ }^{147} \mathrm{Nd}$, see Fig. 13. As can be seen, in several instances these data are discrepant and our procedure was as follows.

In the unresolved resonance region, our evaluations are based on the precise measurements by Wisshak et al. [16] that are generally considered to be the most reliable. In the fast neutron region, our evaluations are guided the EMPIRE code predictions. The Generalized Fermi Liquid (GFL) $\gamma$-ray strength function, as proposed by Mughabghab and Dunford [71], with the Giant Dipole parameters taken from RIPL-2 were used for these predictions. The parameters of the Gilbert-Cameron level densities were calculated from the internal systematics in the EMPIRE code. Such model calculations were normalized to the Wisshak's data in the unresolved resonance region. The experimental data on ${ }^{146,148,150} \mathrm{Nd}$ extend above $1 \mathrm{MeV}$ and are discrepant, but our procedure is supported by the data measured by Trofimov et al. [19] that show consistency with the model calculations.

\section{$5.3 .5(n, 2 n)$ cross sections}

Fig. 14 illustrates our results first by showing ${ }^{142,144} \mathrm{Nd}(\mathrm{n}, 2 \mathrm{n})$ cross sections where experimental data are available. Comparison is made to the ENDF/BVI.8, JEFF-3.1 and JENDL-3.3 evaluations and to the measurements. The JEFF-3.1 evaluations show unphysical high energy behavior due to the neglect of the $(\mathrm{n}, 3 \mathrm{n})$ reaction. Our evaluations on ${ }^{142,144} \mathrm{Nd}$ are guided by the direct neutron counting measurement of Frehaut et al. [54]. Theses results are consistent with the recent experiments by Kasugai et al. [21] on ${ }^{142} \mathrm{Nd}$. We decided to disregard the old activation measurement on ${ }^{142} \mathrm{Nd}$ [72], because it is persistently higher than other measurements.

Fig. 14 further illustrates our results on odd neodymium isotopes, ${ }^{143,145} \mathrm{Nd}$, that rely on the model calculations since no experimental data are available. The parameters used in these calculations were constrained by the (n,2n) measurements on other isotopes as well as by analysis of other reactions on various isotopes of neodymium. Such consistent parametrization justifies appreciable level of confidence in the model predictions. 

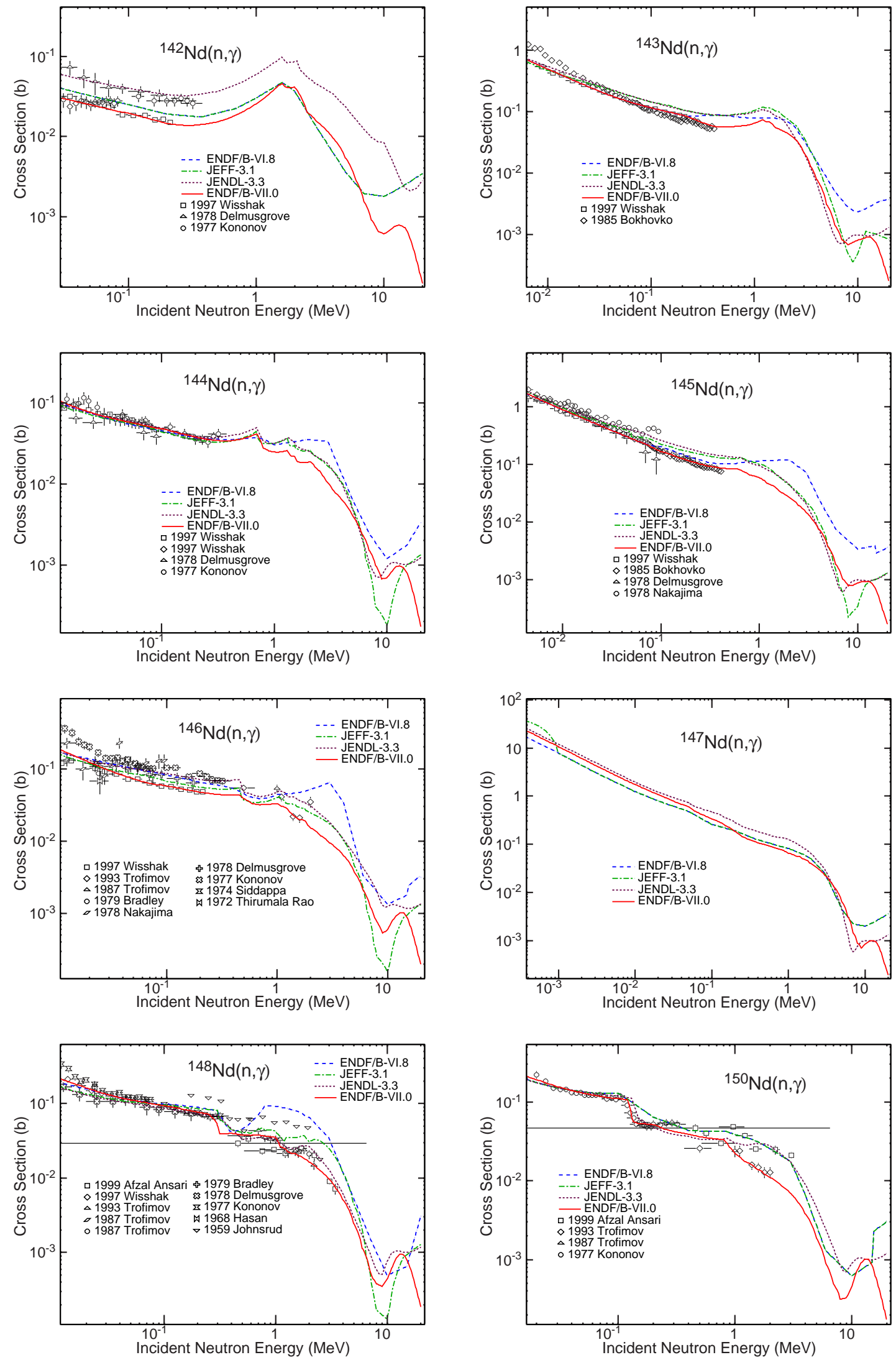

Fig. 13. Evaluated fast neutron capture cross sections on Nd isotopes compared to other evaluations and measurements $[16,17,38,19,18,39,41,67,68,20,42,69,70]$. 

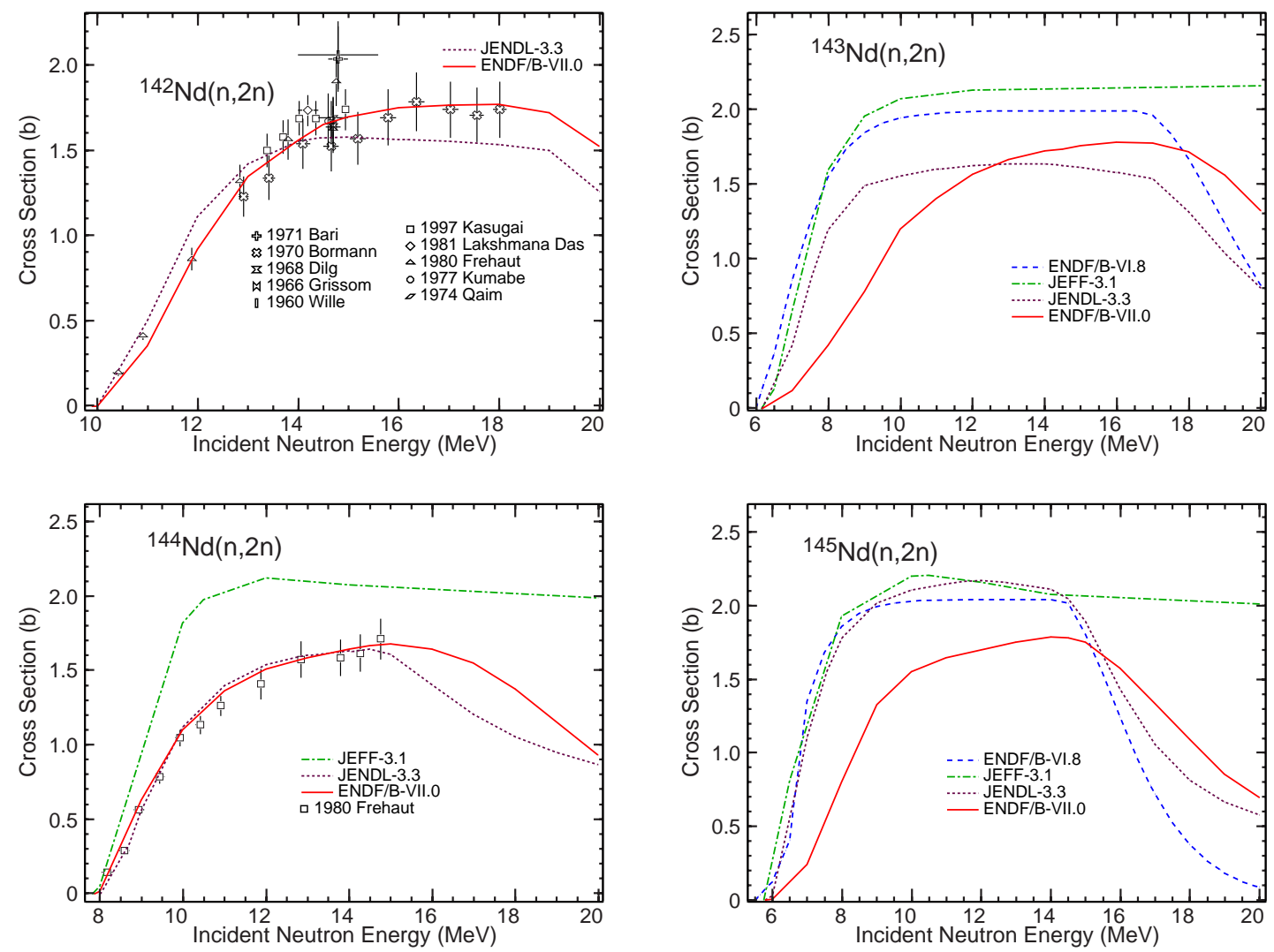

Fig. 14. Evaluated $(\mathrm{n}, 2 \mathrm{n})$ cross sections for all $\mathrm{Nd}$ isotopes compared to other evaluations and to the measurements $[62,59-61,72,54]$.
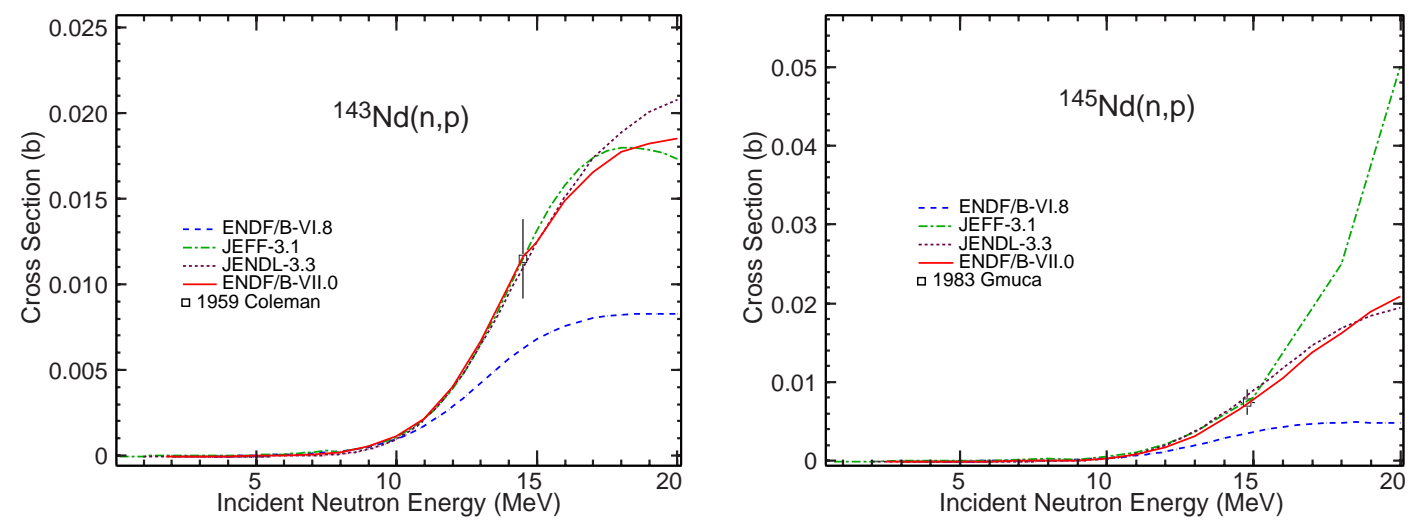

Fig. 15. Evaluated (n,p) cross sections for ${ }^{143,145} \mathrm{Nd}$ isotopes compared to other evaluations and to the measurements of Refs. [73,22].

\subsection{6 (n,p) and $(n, \alpha)$ cross sections}

Fig. 15 illustrates our (n,p) evaluations by showing cross sections on ${ }^{143,145} \mathrm{Nd}(\mathrm{n}, \mathrm{p})$. Our results are compared to the ENDF/B-VI.8, JEFF-3.1 and JENDL-3.3 evaluations and to the measurements $[73,22]$. The evaluations for which there are no experimental data, such as ${ }^{147,150} \mathrm{Nd}$, are based on the model calcula- 

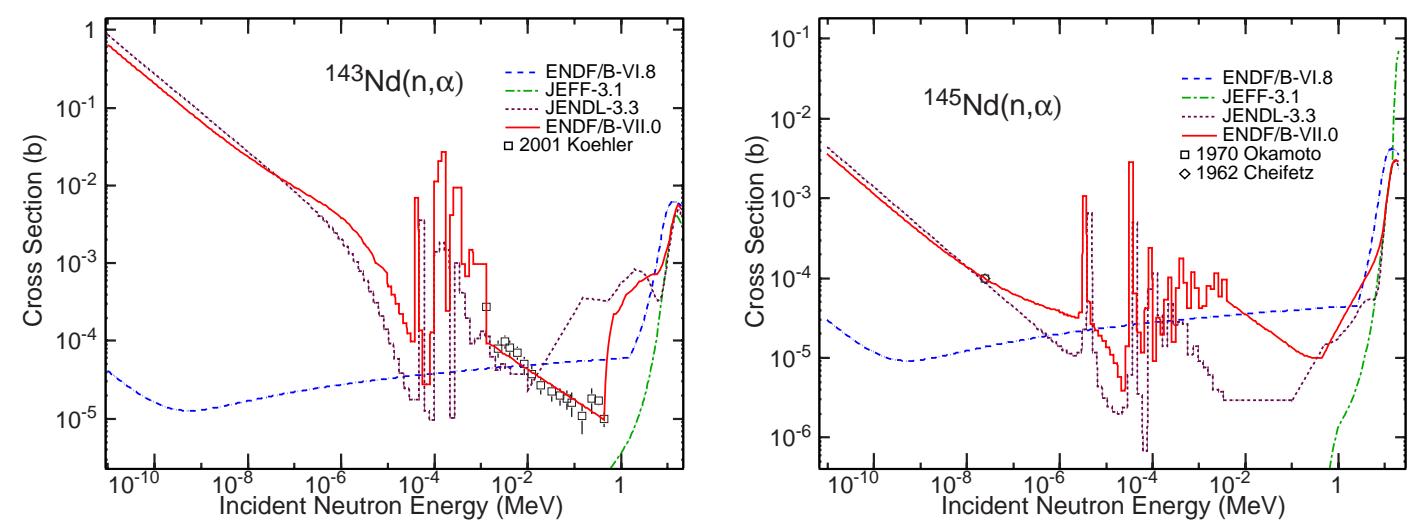

Fig. 16. Evaluated $(\mathrm{n}, \alpha)$ cross sections for ${ }^{143,145} \mathrm{Nd}$ isotopes compared to other evaluations and to the measurements reported $[27,74,75]$.

tions with the consistent set of parameters. In general, $(n, p)$ evaluations are adjusted to reproduce the data measured around $15 \mathrm{MeV}$, therefore, in this energy range the evaluations tend to approach each other. At other energies, especially around $20 \mathrm{MeV}$, the evaluations differ considerably as a result of the differences in modeling adopted by the evaluators. This is also true for $(\mathrm{n}, \alpha)$ cross sections.

Fig. 16 illustrates our (n, $\alpha$ ) evaluations on the case of ${ }^{143,145} \mathrm{Nd}$ isotopes. The $\mathrm{Q}$-values for $(\mathrm{n}, \alpha)$ reactions are positive for all $\mathrm{Nd}$ isotopes and are particularly high for ${ }^{143,145} \mathrm{Nd}, 9.722$ and $8.747 \mathrm{MeV}$, respectively. The measurements by Koehler et al. [27] in the unresolved resonance range on ${ }^{143} \mathrm{Nd}$ and those by Okamoto et al. [74] and Cheifetz et al. [75] of ${ }^{145} \mathrm{Nd}(\mathrm{n}, \alpha)$ at the thermal energy, indicate that $(\mathrm{n}, \alpha)$ cross sections below $1 \mathrm{MeV}$ are small but not negligible. When accounting for this region, we attempted to include an expected resonance structure by assuming that the $(\mathrm{n}, \alpha)$ excitation function is modulated in the same manner as the total cross sections. Accordingly, we adopted shape of the total cross section and scaled it by an appropriate factor to reproduce experimental cross sections. Then, the resonance region was collapsed into 20 energy-groups and stored in the ENDF-6 format (MF=3 file). In the case of ${ }^{143} \mathrm{Nd}$ a factor of $3.91 \times 10^{-5}$ has been applied to obtain $16.49 \mathrm{mb}$ at the thermal energy and reproduce experimental data of Ref. [27] in the unresolved resonance region. For ${ }^{145} \mathrm{Nd}$, we scaled the total cross sections by a factor of $1.4 \times 10^{-6}$ in order to reproduce the thermal value of $0.0001 \mathrm{mb}$ reported in Refs. $[74,75]$.

\subsection{7 $\gamma$-ray and neutron spectra}

Fig. 17 shows $\gamma$-ray emission spectra at $0.0253 \mathrm{eV}$ (thermal), $0.5 \mathrm{MeV}$ and 14.2 $\mathrm{MeV}$ incident neutrons for the ${ }^{143,145} \mathrm{Nd}$ isotopes identified as high priority fission products. The thermal spectrum was obtained by unit-base extrapolation 

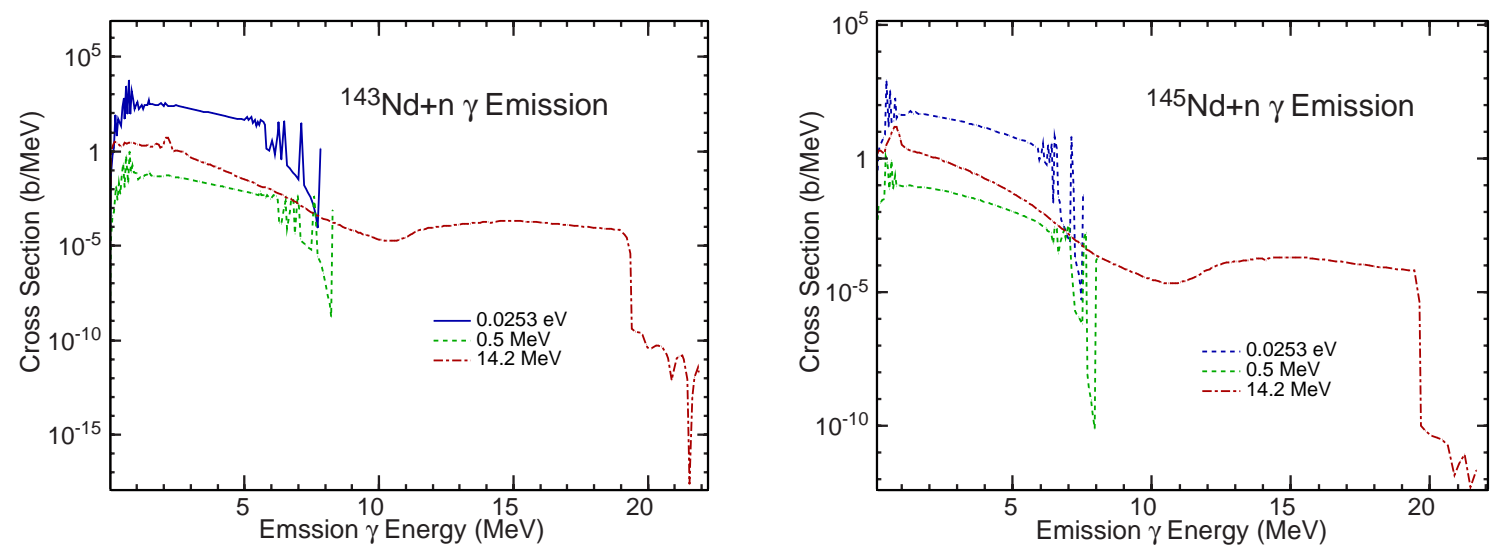

Fig. 17. Evaluated $\gamma$ emission spectra for ${ }^{143,145} \mathrm{Nd}$ at neutron incident energies of $0.0253 \mathrm{eV}, 0.5 \mathrm{MeV}$ and $14.2 \mathrm{MeV}$.
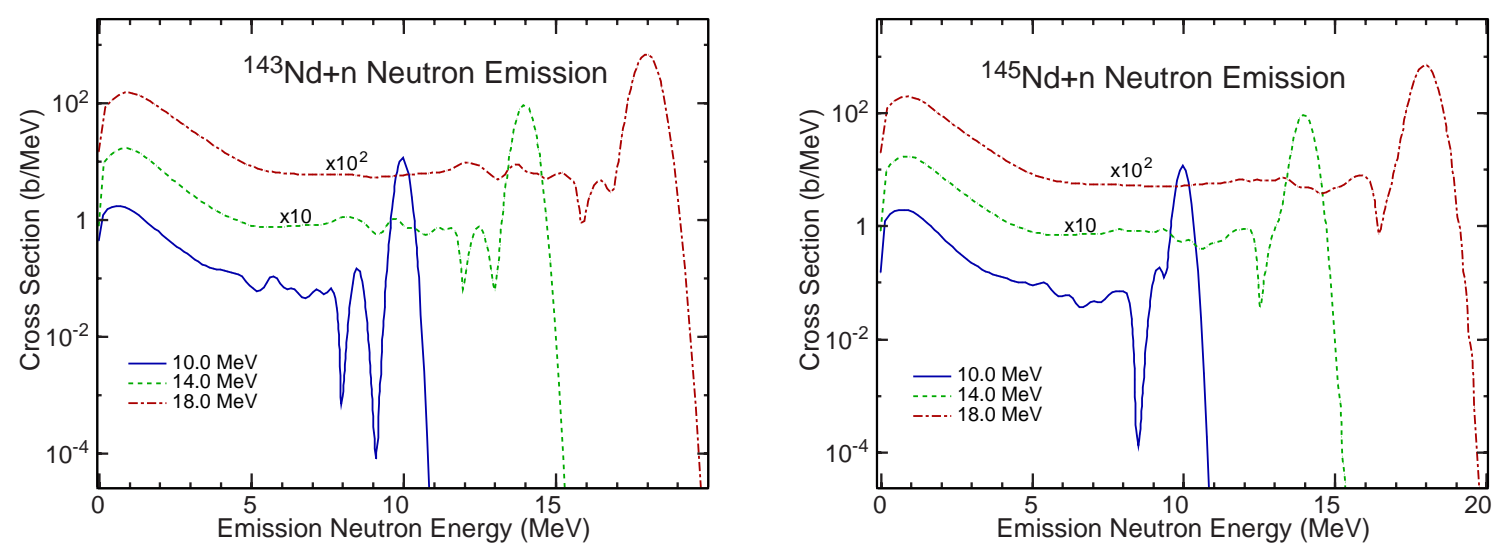

Fig. 18. Evaluated neutron emission spectra for ${ }^{143,145} \mathrm{Nd}$ at neutron incident energies of 10,14 and $18 \mathrm{MeV}$. The lines were scaled up by the factors indicated in the figure.

of EMPIRE calculations at the lowest energy down to the thermal energy. The spectra at $0.0253 \mathrm{eV}$ and $0.5 \mathrm{MeV}$ were calculated within the Hauser-Feshbach statistical model with the GFL $\gamma$-ray strength function and Gilbert-Cameron level densities. At higher energies, the preequilibrium mechanism was included in the calculations. Its contribution is evident in the $14.2 \mathrm{MeV}$ spectrum where it forms a plateau for $\gamma$-ray energies above $10 \mathrm{MeV}$. The pure compound nucleus predictions in this energy range would be orders of magnitude lower.

A few examples of neutron emission spectra are shown in Fig. 18. We selected high priority fission products, ${ }^{143,145} \mathrm{Nd}$, and incident neutrons of 10 , 14 and $18 \mathrm{MeV}$ to illustrate role of the preequilibrium mechanism. A smooth bump at the low emission energies is formed by the multiple (if energetically possible) emissions from the fully equilibrated compound nucleus. The relatively flat part above $5 \mathrm{MeV}$ is dominated by the preequilibrium mechanism. Marked fluctuations within this region result from the population of collective 

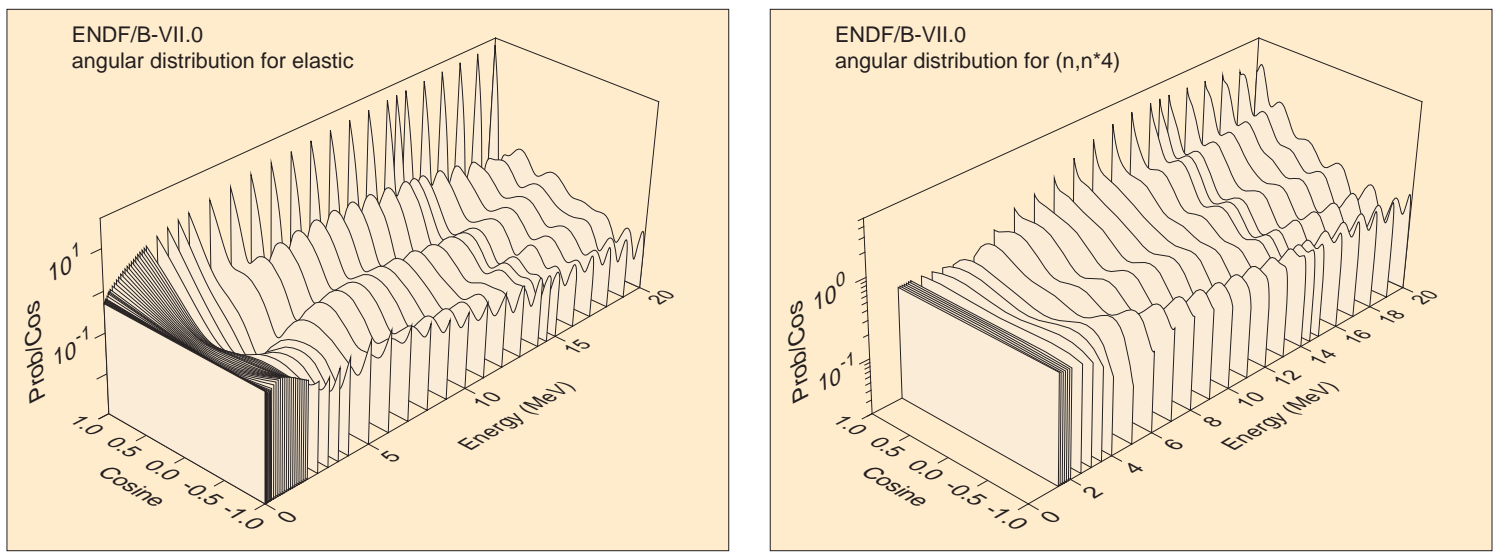

Fig. 19. Angular distributions for elastic (left plot) and inelastic (right plot) scattering of neutrons on ${ }^{143} \mathrm{Nd}$ at different incident energies.

levels in the continuum through the MSD and DWBA mechanisms. Coupledchannels complete the spectrum with transitions to discrete levels including elastic peak.

\subsubsection{Energy-angle correlated cross sections}

The present evaluations also include a full set of double-differential cross sections coded in the ENDF-6 format as File 6. As an example, Fig. 19 shows angular distributions for neutron elastic and inelastic scattering on ${ }^{143} \mathrm{Nd}$ for different incident neutron energies. Both distributions are isotropic, rather than symmetric, at low incident energies. This approximation results from the lack of angular distribution capabilities in the current implementation of the statistical model in the EMPIRE code. At incident energies above $2 \mathrm{MeV}$, the distributions become forward peaked and reveal diffraction pattern typical for the optical model.

\section{Conclusions}

New neutron cross section evaluations for a complete chain of neodymium isotopes were performed in the neutron-incident energy range from $10^{-5} \mathrm{eV}$ to $20 \mathrm{MeV}$. This work includes the revision of thermal and resonance data recently published in the Atlas of Neutron Resonances, and the fast neutron region based on the nuclear reaction model code EMPIRE-2.19.

The low energy region was carefully assessed in order to ensure adequate reproduction of the integral data such as the thermal capture cross sections and the resonance integrals as well as to reproduce microscopic data in the 
unresolved resonance region.

In the fast neutron range, we cover cross sections for almost all reaction channels such as total, elastic scattering, inelastic scattering, capture, and particle emission cross sections, and include photon production, energy spectra of emitted particles and their angular distributions. Since the complete chain of neodymium isotopes was evaluated simultaneously, we were in a position to establish a consistent set of model parameters constrained by the entirety of experimental data available for neodymiums. This approach greatly improves confidence in the model predictions for the isotopes for which measurements are insufficient or nonexistent.

The evaluated data were converted into ENDF-6 formatted files, checked by a set of checking codes, processed with the Monte Carlo code NJOY-99.161, and subject to test runs with the code MCNP5 to ensure that the files can be used in transport calculations. All evaluations were adopted by the new U.S. evaluated library, ENDF/B-VII.0, released in December 2006.

\section{Acknowledgments}

One of us (HIK) is most grateful to the staff of the National Nuclear Data Center for the warm hospitality and support provided during his visits to BNL when working on this project. This work has been performed in part under the auspices of the Korean Ministry of Science and Technology as the longterm R\&D project. Work at Brookhaven National Laboratory was sponsored by the Office of Nuclear Physics, Office of Science of the U.S. Department of Energy under Contract No. DE-AC02-98CH10886 with Brookhaven Science Associates, LLC.

\section{References}

[1] H. I. Kim, Y.-O. Lee, M. Herman, S. F. Mughabghab, P. Obložinský, D. Rochman, Evaluation of neutron induced reactions for 32 fission products, Tech. Rep. BNL-77775-2007-IR, Brookhaven National Laboratory (2007).

[2] M. B. Chadwick, P. Obložinský, M. Herman, N. M. Greene, R. D. McKnight, D. L. Smith, P. G. Young, R. E. MacFarlane, G. M. Hale, S. C. Frankle, A. C. Kahler, T. Kawano, R. C. Little, D. G. Madland, P. Moller, R. D. Mosteller, P. R. Page, P. Talou, H. Trellue, M. C. White, W. B. Wilson, R. Arcilla, C. L. Dunford, S. F. Mughabghab, B. Pritychenko, D. Rochman, A. A. Sonzogni, C. R. Lubitz, T. H. Trumbull, J. P. Weinman, D. A. Brown, D. E. Cullen, D. P. Heinrichs, D. P. McNabb, H. Derrien, M. E. Dunn, N. M. Larson, L. C. 
Leal, A. D. Carlson, R. C. Block, J. B. Briggs, E. T. Cheng, H. C. Huria, M. L. Zerkle, K. S. Kozier, A. Courcelle, V. Pronyaev, S. C. van der Marck, ENDF/BVII.0: Next generation evaluated nuclear data library for nuclear science and technology, Nucl. Data Sheets 107 (2006) 2931.

[3] M. D. DeHart, Sensitivity and parametric evaluations of significant aspects of burnup credit for PWR spent fuel package, Tech. Rep. ORNL/TM-12973, Oak Ridge National Laboratory (1995).

[4] S. Oh, J. Chang, S. F. Mughabghab, Neutron cross section evaluations of fission products below the fast neutron region, Tech. Rep. BNL-NCS-67469, Brookhaven National Lab. (2000).

[5] S. F. Mughabghab, D. Garber, Neutron Cross Sections: Resonance Parameters, Vol. 1, BNL-325, 3rd Edition, 1973.

[6] P. Moldauer, Optical model of low energy neutron interactions with spherical nuclei, Nuclear Physics 47 (1963) 65.

[7] S. F. Mughabghab, M. Divadeenam, N. E. Holden, Neutron Cross Sections: $\mathrm{Z}=1-60$, Vol. 1A, Academic Press, New York, 1981.

[8] H. Tellier, Properties of levels induced in stable isotopes of neodymium by neutrons in the resonance region (in French), Tech. Rep. 1459, Centre d'Études Nucléaires (1971).

[9] A. R. Delmusgrove, B. J. Allen, J. W. Boldeman, R. L. Macklin, Tech. Rep. 401, Australian AEC Reports (1977).

[10] V. Benzi, G. Reffo, Report CCDN-NW/10 (1969).

[11] S. F. Mughabghab, Atlas of Neutron Resonances: Thermal Cross Sections and Resonance Parameters, Elsevier, Amsterdam, 2006.

[12] M. Herman, R. Capote, B. Carlson, P. Obložinský, M. Sin, A. Trkov, H. Wienke, V. Zerkin, EMPIRE: Nuclear Reaction Model Code System for Data Evaluation, Nucl. Data Sheets 108 (2007) 2655.

[13] M. Herman, R. Capote, B. Carlson, P. Obložinský, M. Sin, A. Trkov, V. Zerkin, EMPIRE nuclear reaction model code, version 2.19 (Lodi), www.nndc.bnl.gov/empire219/ (March 2005).

[14] P. Young, M. Herman, P. Obložinský, T. Belgya, O. Bersillon, R. Capote, T. Fukahori, G. Zhigang, S. Goriely, A. Ignatyuk, S. Kailas, A. Koning, V. Plujko, Handbook for calculations of nuclear reaction data, RIPL-2, TECDOC-1506, IAEA, Vienna (2006).

[15] W. P. Poenitz, J. F. Whalen, Neutron total cross section measurements in the energy region from $47 \mathrm{keV}$ to $20 \mathrm{MeV}$, Tech. Rep. 80, Argonne National Laboratory (1983).

[16] K. Wisshak, F. Voss, F. Käppeler, K. Guber, L. Kazakov, G. Reffo, Tech. Rep. 5967, Kernforschungszentrum Karlsruhe (1997). 
[17] M. V. Bokhovko, L. E. Kazakov, V. N. Kononov, E. D. Poletaev, V. M. Timokhov, A. A. Voevodskiy, Radiative capture cross-section measurement for Nd-143 and Nd-145 isotopes in the energy range $4-420 \mathrm{keV}$, Vop. At. Nauki i Tekhn., Ser. Yadernye Konstanty (3) (1985) 12.

[18] Y. N. Trofimov, Activation cross-sections for 33 nuclei at the neutron energy 2 MeV, Vop. At. Nauki i Tekhn., Ser. Yadernye Konstanty (4) (1987) 10.

[19] Y. N. Trofimov, Neutron radiation capture cross-sections for even neodimium isotopes in the energy range $0.5-2.0 \mathrm{MeV}$, Vop. At. Nauki i Tekhn., Ser. Yadernye Konstanty (1) (1993) 17.

[20] M. Afzal Ansari, R. K. Y. Singh, R. P. Gautam, S. Kailas, Fast neutron radiative capture cross-sections in fission product isotopes of neodymium, Annals of Nuclear Energy 26 (1999) 553.

[21] Y. Kasugai, Y. Ikeda, Y. Uno, Activation cross section measurement for La, Ce, Pr, Nd, Gd, Dy and Er isotopes by $14 \mathrm{MeV}$ neutrons, in: Conf. on Nucl. Data for Sci. and Techn., Trieste, Vol. 1, 1997, p. 635.

[22] S. Gmuca, I. Ribanský, Neutron activation cross sections on Nd isotopes at 14.8 MeV, Acta Physica Slovaca 33 (1983) 9.

[23] A. J. Do, J. Dresler, U. Garuska, M. Herman, A. Marcinkowski, Cross section of the $(\mathrm{n}, 2 \mathrm{n})$ reactions on Ba-134, Nd-142, Nd-150 and Sm-144, Jour. of Physics G10 (1984) 91.

[24] A. Grallert, J. Csikai, C. M. Buczko, I. Shaddad, Investigations on the systematics in $(\mathrm{n}, \alpha)$ cross sections at $14.6 \mathrm{MeV}$, Tech. Rep. 286, IAEA (1993).

[25] H. Sakane, T. Iida, A. Takahashi, H. Yamamoto, K. Kawade, Measurement of cross sections producing short-lived nuclei by $14 \mathrm{MeV}$ neutrons - Cd, Sn, Te, Nd, Gd, Re, Conference Report 98-003, JAERI (1997).

[26] A. Bari, $14.8 \mathrm{MeV}$ neutron activation cross sections for $(\mathrm{n}, \mathrm{p})$ and $(\mathrm{n}, \alpha)$ reactions for some rare-earth nuclides, J. of Radioanalytical Chemistry 75 (1982) 189.

[27] P. E. Koehler, Y. M. Gledenov, J. Andrzejewski, K. H. Guber, S. Raman, T. Rauscher, Improving explosive nucleosynthesis models via $(\mathrm{n}, \alpha)$ measurements, Nuclear Physics A688 (2001) 86.

[28] A. D. Majeddin, V. Semkova, R. D. C. M. Buczko, J. Csikai, Investigations on $(\mathrm{n}, \alpha)$ cross sections in the $14 \mathrm{MeV}$ region, private communication (1997).

[29] S. F. Mughabghab, Neutron Cross Sections: Z=61-100, Vol. 1B, Academic Press, New York, 1984.

[30] M. Lucas, R. Hagemann, R. Naudet, C. Renson, C. Chevalier, Determination by irradiation in the triton reactor of neutron capture cross sections for isotopes involved in the Oklo phenomenon (in French with abstract in English), Vol. 1, 1977, p. 431, meeting on Natural Fission Reactors, Paris 1977. 
[31] V. P. Vertebnyj, N. L. Gnidak, E. A. Pavlenko, The neutron cross section of isotopes of neodymium, Tech. Rep. 17, Yaderno-Fizicheskie Issledovaniya Reports (1974).

[32] M. J. Cabell, M. Wilkins, Mass spectrometric measurements of the neutron capture cross sections of Nd-142, Nd-143, Nd-144 and Nd-145 for reactor and Maxwellian neutrons, Journal of Inorganic and Nuclear Chemistry 30 (1968) 897.

[33] R. B. Tattersall, H. Rose, S. K. Pattenden, D. Jowitt, Pile oscillator measurements of resonance absorption integrals, J. of Nuclear Energy, Part A $12(1960) 32$.

[34] H. J. Hay, The slow neutron cross section of Nd-143, Journal of Nuclear Energy 7 (1958) 199.

[35] N. J. Pattenden, Some neutron cross sections of importance to reactors - Tc99, Nd-143, Nd-145, Sm-149, Sm-152, Eu-151, Eu-153, Gd-155, Gd-157, Pu-240, Vol. 16, 1958, p. 44, 2nd Internat. At. En. Conf., Geneva 1958.

[36] K. Wisshak, F. Voss, F. Kappeler, L. Kazakov, G. Reffo, Stellar neutron capture cross sections of the Nd isotopes, Physical Review C 57 (1998) 391.

[37] V. N. Kononov, E. D. Poletaev, B. D. Yurlov, M. V. Bokhovko, L. E. Kazakov, V. M. Timokhov, Fast neutron radiative capture cross-sections and d-wave strength functions, 1981, 4th Symp. Neutr. Capt. Gamma-Ray Spectroscopy, Grenoble.

[38] A. R. D. Musgrove, B. J. Allen, J. W. Boldeman, R. L. Macklin, Non-statistical effects in the radiative capture cross- sections of the neodymium isotopes, in: Int. Conf. on Neutr. Phys. and Nucl. Data, Harwell, 1978, p. 449.

[39] Y. Nakajima, A. Asami, Y. Kawarasaki, Y. Furuta, Neutron capture cross section measurements of Nd-143, Nd-145, Nd-146 and Nd-148, in: Int. Conf. on Neutr. Phys. and Nucl. Data, Harwell, 1978, p. 438.

[40] R. W. Hockenbury, H. D. Knox, N. N. Kaushal, Fission product capture cross sections in the keV region, in: Conf. on Nucl. Cross-Sect. and Techn., Washington, Vol. 2, 1975, p. 905.

[41] V. N. Kononov, B. D. Jurlov, E. D. Poletaev, V. M. Timokhov, G. N. Manturov, Average neutron radiative capture cross-section in the energy range $5-30 \mathrm{keV}$ for In, Ta, Au and Nd, Sm, Eu, Gd, Er isotopes, Tech. Rep. 22, Yadernye Konstanty (1977).

[42] T. Bradley, Z. Parsa, M. L. Stelts, R. E. Chrien, Stellar nucleosynthesis and the 24-keV neutron capture cross sections of some heavy nuclei, in: Conf.o n Nuclear Cross Sections for Techn., Knoxville, 1979, p. 344.

[43] R. E. Shamu, E. M. Bernstein, J. J. Ramirez, C. H. Lagrange, Effects of deformation on neutron total cross sections of even-A Nd and Sm isotopes, Physical Review C22 (1980) 1857. 
[44] A. N. Djumin, A. I. Egorov, G. N. Popova, V. A. Smolin, Nuclear deformation influence on total neutron cross-sections, Izv. Rossiiskoi Akademii Nauk, Ser. Fiz. 37 (5) (1973) 1019.

[45] G. Haouat, J. Lachkar, C. Lagrange, M. T. Mcellistrem, Y. Patin, R. E. Shamu, J. Sigaud, Optical potential and nuclear deformation for even $\mathrm{Nd}$ isotopes from fast neutron scattering, Physical Review C20 (1979) 78.

[46] D. G. Foster Jr, D. W. Glasgow, Neutron total cross sections, 2.5 - $15 \mathrm{MeV}$. I. Experimental, Physical Review C3 (1971) 576.

[47] J. Haugsnes, Ph.D. thesis (1967).

[48] K. K. Seth, R. H. Tabony, E. G. Bilpuch, H. W. Newson, Neutron total cross sections and intermediate resonances, Physics Letters 16 (1965) 306.

[49] J. P. Conner, Total neutron cross sections near $14.1 \mathrm{MeV}$, Physical Review 109 (1958) 1268.

[50] A. Okazaki, S. E. Darden, R. B. Walton, Total cross section of rare earths for fast neutrons, Physical Review 93 (1954) 461.

[51] A. J. Koning, J. P. Delaroche, Local and global nucleon optical models from 1 keV to $200 \mathrm{MeV}$, Nuclear Physics A713 (2003) 231.

[52] V. Avrigeanu, P. E. Hodgson, M. Avrigeanu, Global optical potentials for emitted alpha particles, Physical Review C49 (1994) 2136.

[53] R. O. Owens, J. H. Towle, The level density of the deformed rare-earth nuclei, Nuclear Physics A112 (1968) 337.

[54] J. Frehaut, A. Bertin, R. Bois, J. Jary, Status of $(n, 2 n)$ cross section measurements at Bruyères-le-Châtel, private communication (1980).

[55] S. L. Sothras, G. N. Salaita, (n,2n) cross sections at 14.8-MeV on some closed shell nuclides, Journal of Inorganic and Nuclear Chemistry 40 (1978) 585.

[56] I. Kumabe, E. Kotake, F. Nagahama, Activation cross sections for (n,2n) reaction on neodynium, samarium, gadolinium and ytterbium at $14.6 \mathrm{MeV}$, J. of Nuclear Science and Technology 14 (1977) 319.

[57] N. Lakshmana Das, C. V. Srinivase Rao, B. V. Thirumala Rao, J. Rama Rao, Pre-compound decay in (n,2n) reactions at $14.2 \mathrm{MeV}$, in: 17th Nucl. and Solid State Physics Symp., Bombay, Vol. 2, 1974, p. 105.

[58] S. M. Qaim, Total (n,2n) cross sections and isomeric cross-section ratios at 14.7 $\mathrm{MeV}$ in the region of rare earths, Nuclear Physics A224 (1974) 319.

[59] M. Bormann, H. H. Bissem, E. Magiera, R. Warnemunde, Total cross sections and isomeric cross section ratios for $(\mathrm{n}, 2 \mathrm{n})$ reactions in the energy region 12 $18 \mathrm{MeV}$, Nuclear Physics A157 (1970) 481.

[60] W.

Dilg, H. Vonach, G. Winkler, P. Hille, Messing von (n,2n) Wirkungsquerschnitten an Schweren Kernen, Nuclear Physics A118 (1968) 9. 
[61] J. T. Grissom, D. R. Koehler, W. L. Alford, Nd141 and its production in the (n,2n) reaction, Physical Review 142 (1966) 725.

[62] A. Bari, 14.8 MeV neutron activation cross sections of rubidium, strontium, zirconium, niobium and rare-earth nuclides, Ph.D. thesis (1971).

[63] P. Rama Prasad, J. Rama Rao, E. Kondaiah, Cross sections for (n,2n), (n, $\alpha$ ) and $(\mathrm{n}, \mathrm{p})$ reactions in rare earth isotopes at $14.2 \mathrm{MeV}$, Nuclear Physics A125 (1969) 57.

[64] M. P. Menon, M. Y. Cuypers, 14.5-MeV neutron activation cross section for some of the rare-earth nuclides and their relation to the nuclear shell structure, Physical Review 156 (1967) 1340.

[65] D. F. Coope, S. N. Tripathi, M. C. Schell, M. T. Mc Ellistrem, Neutron scattering from spherical and deformed Nd isotopes, Bulletin of the American Physical Society 24 (1979) 854.

[66] M. C. Schell, A low energy neutron scattering study on $146 \mathrm{Nd}$ and $150 \mathrm{Nd}$, Ph.D. thesis (1976).

[67] K. Siddappa, M. Sriramachandra Murty, J. Rama Rao, Neutron activation cross-sections in rare earths and heavier nuclei, Annals of Physics (New York) 83 (1974) 355.

[68] B. V. Thirumala Rao, J. Rama Rao, E. Kondaiah, Neutron capture cross sections at $25 \mathrm{keV}$, Jour. of Physics A5 (1972) 468.

[69] S. S. Hasan, A. K. Chaubey, M. L. Sehgal, Neutron activation cross-sections at $24 \mathrm{keV}$, Nuovo Cimento B 58 (1968) 402.

[70] A. E. Johnsrud, M. G. Silbert, H. H. Barschall, Energy dependence of fastneutron activation cross section, Physical Review 116 (1959) 927.

[71] S. F. Mughabghab, C. L. Dunford, A dipole-quadrupole interaction term in E1 photon transitions, Phys. Lett. B 487 (2000) 155.

[72] R. G. Wille, R. W. Fink, Activation cross sections for 14.8-MeV neutrons and some new radioactive nuclides in the rare earth region, Physical Review 118 (1960) 242 .

[73] R. F. Coleman, B. E. Hawker, L. P. O‘Connor, J. L. Perkin, Cross sections for $(\mathrm{n}, \mathrm{p})$ and $(\mathrm{n}, \alpha)$ reactions with $14.5 \mathrm{MeV}$ neutrons, in: Proceedings of the Physical Society (London), Vol. 73, 1959, p. 215.

[74] K. Okamoto, The (n, $\alpha$ ) reaction on samarium and neodymium isotopes induced by thermal neutrons, Nuclear Physics A141 (1970) 193.

[75] E. Cheifetz, J. Gilat, A. F. Yavin, S. G. Cohen, Alpha-particle emission competing with thermal neutron capture gamma-rays in rare earth isotopes, Physics Letters 1 (1962) 289. 